\title{
Gradient schemes: a generic framework for the discretisation of linear, nonlinear and nonlocal elliptic and parabolic equations
}

\author{
Jérôme Droniou ${ }^{1}$, Robert Eymard ${ }^{2}$, Thierry Gallouët ${ }^{3}$, Raphaèle Herbin ${ }^{4}$.
}

5 November 2012

\begin{abstract}
Gradient schemes are nonconforming methods written in discrete variational formulation and based on independent approximations of functions and gradients, using the same degrees of freedom. Previous works showed that several well-known methods fall in the framework of gradient schemes. Four properties, namely coercivity, consistency, limit-conformity and compactness, are shown in this paper to be sufficient to prove the convergence of gradient schemes for linear and nonlinear elliptic and parabolic problems, including the case of nonlocal operators arising for example in image processing. We also show that the Hybrid Mimetic Mixed family, which includes in particular the Mimetic Finite Difference schemes, may be seen as gradient schemes meeting these four properties, and therefore converges for the class of above mentioned problems.
\end{abstract}

\section{Introduction}

We consider general elliptic equations of the form

$$
\begin{aligned}
& -\operatorname{div} \boldsymbol{a}(\boldsymbol{x}, \bar{u}, \nabla \bar{u})=f \text { in } \Omega, \\
& \bar{u}=0 \text { on } \partial \Omega
\end{aligned}
$$

where $\Omega$ is an open bounded connected subset of $\mathbb{R}^{d}$, for $d \in \mathbb{N}^{*}$ with a boundary denoted by $\partial \Omega=\bar{\Omega} \backslash \Omega$. The solution $\bar{u}$ is sought in the space $W_{0}^{1, p}(\Omega)$ for some $p \in(1,+\infty)$. Particular choices of $\boldsymbol{a}$ include general anisotropic heterogeneous linear operators $\boldsymbol{a}(\boldsymbol{x}, u, \boldsymbol{\xi})=\Lambda(\boldsymbol{x}) \boldsymbol{\xi}$, Leray-Lions operators such as the $p$ Laplacian $\boldsymbol{a}(\boldsymbol{x}, u, \boldsymbol{\xi})=|\boldsymbol{\xi}|^{p-2} \boldsymbol{\xi}$, and some nonlinear and nonlocal diffusion operators $\boldsymbol{a}(\boldsymbol{x}, u, \boldsymbol{\xi})=\Lambda(u, \boldsymbol{x}) \boldsymbol{\xi}$ for $u$ in a given functional space.

We shall also consider the evolution problem associated to problem (1.1), which is the following nonlinear parabolic problem (where $T \in(0,+\infty))$ :

$$
\begin{aligned}
& \partial_{t} \bar{u}-\operatorname{div} \boldsymbol{a}(\boldsymbol{x}, \bar{u}, \nabla \bar{u})=f \text { in } \Omega \times(0, T), \\
& \bar{u}(\boldsymbol{x}, 0)=u_{\text {ini }}(\boldsymbol{x}) \text { in } \Omega, \\
& \bar{u}=0 \text { on } \partial \Omega \times(0, T) .
\end{aligned}
$$

Such evolution equations, involving non local operators, arise in particular in image processing, in the spirit of $[10,14,29]$ and references therein. The linear anisotropic heterogeneous case is involved in most models used in underground engineering (oil recovery, nuclear waste disposals, etc.). In these models, computations have to be performed on meshes adapted to the geological layers, and including complex geometrical features such as faults, vanishing layers, inclined wells, highly heterogeneous permeability fields, local nonconforming refinement. Since standard finite element methods are not well adapted to such constraints, a large number of schemes have been developed for the numerical approximation of (1.1) and (1.2) in this case. Although we cannot give here an exhaustive list, let us mention a few of them:

- the Multi-Point Flux Approximation (MPFA) schemes [1],

\footnotetext{
${ }^{1}$ School of Mathematical Sciences, Monash University, Victoria 3800, Australia. email: jerome.droniou@monash.edu

${ }^{2}$ Université Paris-Est, Laboratoire d'Analyse et de Mathématiques Appliquées, UMR 8050, 5 boulevard Descartes, Champs-sur-Marne, 77454 Marne-la-Vallée Cedex 2, France. email:Robert.Eymard@univ-mlv.fr

${ }^{3}$ L.A.T.P., UMR 6632, Université de Provence, gallouet@cmi.univ-mrs.fr

${ }^{4}$ L.A.T.P., UMR 6632, Université de Provence, herbin@cmi.univ-mrs.fr
} 
- the Hybrid Mimetic Mixed family which includes the Mimetic Finite Difference schemes, the SUSHI scheme and the Mixed Finite Volume scheme, see [18] and references therein,

- the Discrete Duality Finite Volume (DDFV) schemes [25, 13, 5].

A construction and proof of convergence in the case of nonlinear Leray-Lions operators is already known for some of these methods, namely the Mixed Finite Volume method [15], the DDFV scheme [2], the SUSHI scheme in its cell centred version [19] (see also [6] for a Discontinuous Galerkin scheme for the $p$-Laplacian).

Although the analytical tools used to study these methods are often similar, they are usually considered as different schemes whose study requires new work each time. However, as noticed in [21, 23, 22], many of these methods can be included in the unified theoretical framework of (possibly) nonconforming gradient schemes. In particular, the following methods are gradient schemes:

- some MPFA and DDFV schemes in 2D or 3D,

- the Galerkin methods, including the Conforming Finite Element methods,

- the nonconforming P1 Finite Element discretisation,

- the Mixed Finite Element discretisations.

The aim of this paper is to show that gradient schemes, which can be characterised by a small number of discrete elements, have the two following interesting properties:

1. They provide a generic framework in which only a small number of discrete assumptions is required to establish error estimates for linear stationary equations and convergence proofs for both nonlinear stationary and transient equations.

2. They encompass the entire Hybrid Mimetic Mixed family, and thus in particular the Mimetic Finite Difference methods. Given the success of these methods for linear problems, see e.g. [8, 9, 3, 4], we find quite exciting and remarkable to extend them to fully nonlinear problems and to prove their convergence in this setting.

This paper is organised as follows. In Section 2, we present the small number of discrete elements which are needed to define a gradient scheme. In Section 3, we consider the stationary cases. We provide an error estimate in the linear case, and a convergence proof for Leray-Lions problems including a nonlocal dependency of the operator. In Section 4, we give a convergence proof for the time-dependent LerayLions problem, using a generic discrete Aubin-Lions theorem. A particularly remarkable fact is that these proofs are made under very few and generic discretisation assumptions. Finally, in Section 5, we show that all schemes derived from the Hybrid Mimetic Mixed family are gradient schemes which satisfy the properties under which the convergence analysis of Sections 3-4 are performed. This therefore shows that Hybrid Mimetic Mixed methods are suitable not only for local linear problems but also for nonlocal nonlinear problems.

\section{Gradient discretisations and gradient schemes}

\subsection{Definitions}

We present here properties which are shown in the next sections to be sufficient for the convergence of gradient schemes, considering homogeneous Dirichlet boundary conditions.

A gradient scheme can be viewed as a general formulation of several discretisations of (1.1) which are based on a nonconforming approximation of the weak formulation of the problem. The approximation of the weak formulation of (1.1) is based on some discrete spaces and mappings, the set of which we call a gradient discretisation. Throughout this paper, $\Omega$ is an open bounded subset of $\mathbb{R}^{d}, d \in \mathbb{N}^{\star}$, and $p \in(1,+\infty)$. 
Definition 2.1 (Gradient discretisation) A gradient discretisation $\mathcal{D}$ of Problem (1.1) is defined by $\mathcal{D}=\left(X_{\mathcal{D}, 0}, \Pi_{\mathcal{D}}, \nabla_{\mathcal{D}}\right)$, where:

1. the set of discrete unknowns $X_{\mathcal{D}, 0}$ is a finite dimensional vector space on $\mathbb{R}$,

2. the linear mapping $\Pi_{\mathcal{D}}: X_{\mathcal{D}, 0} \rightarrow L^{p}(\Omega)$ is the reconstruction of the approximate function,

3. the linear mapping $\nabla_{\mathcal{D}}: X_{\mathcal{D}, 0} \rightarrow L^{p}(\Omega)^{d}$ is the discrete gradient operator. It must be chosen such that $\|\cdot\|_{\mathcal{D}}:=\left\|\nabla_{\mathcal{D}} \cdot\right\|_{L^{p}(\Omega)^{d}}$ is a norm on $X_{\mathcal{D}, 0}$.

Remark 2.2 (Boundary conditions.) The definition of $\|\cdot\|_{\mathcal{D}}$ depends on the considered boundary conditions. For simplicity we only consider here homogeneous Dirichlet boundary conditions, but other conditions can easily be addressed. For example, in the case of homogeneous Neumann boundary conditions, we would use the notation $X_{\mathcal{D}}$ instead of $X_{\mathcal{D}, 0}$ for the discrete space, and define $\|\cdot\|_{\mathcal{D}}:=$ $\left(\left\|\Pi_{\mathcal{D}} \cdot\right\|_{L^{p}(\Omega)}^{p}+\left\|\nabla_{\mathcal{D}} \cdot\right\|_{L^{p}(\Omega)^{d}}^{p}\right)^{1 / p}$.

The related gradient scheme is merely the discretisation of the weak formulation of (1.1) obtained by using the discrete space and mappings of the gradient discretisation.

Definition 2.3 (Gradient scheme) If $\mathcal{D}=\left(X_{\mathcal{D}, 0}, \Pi_{\mathcal{D}}, \nabla_{\mathcal{D}}\right)$ is a gradient discretisation, then we define the related gradient scheme for (1.1) by

$$
\begin{aligned}
& \text { Find } u \in X_{\mathcal{D}, 0} \text { such that, } \forall v \in X_{\mathcal{D}, 0} \\
& \int_{\Omega} \boldsymbol{a}\left(\boldsymbol{x}, \Pi_{\mathcal{D}} u, \nabla_{\mathcal{D}} u(\boldsymbol{x})\right) \cdot \nabla_{\mathcal{D}} v(\boldsymbol{x}) \mathrm{d} \boldsymbol{x}=\int_{\Omega} f(\boldsymbol{x}) \Pi_{\mathcal{D}} v(\boldsymbol{x}) \mathrm{d} \boldsymbol{x} .
\end{aligned}
$$

Since $X_{\mathcal{D}, 0}$ is a finite dimensional space, there exists at least one solution to (2.1) provided that $\boldsymbol{a}$ and $f$ satisfy the usual assumptions that ensure the existence of a weak solution to (1.1) (see Section 3). For the solution of this finite dimensional problem to converge to a weak solution of (1.1), some consistency and stability properties are of course required. As in the framework of Finite Element methods, stability is obtained thanks to some uniform coercivity of the discrete operator which relies on a discrete Poincaré inequality.

Definition 2.4 (Coercivity) Let $\mathcal{D}$ be a gradient discretisation for Problem (1.1) in the sense of Definition 2.1, and let $C_{\mathcal{D}}$ be the norm of the linear mapping $\Pi_{\mathcal{D}}$, defined by

$$
C_{\mathcal{D}}=\max _{v \in X_{\mathcal{D}, 0} \backslash\{0\}} \frac{\left\|\Pi_{\mathcal{D}} v\right\|_{L^{p}(\Omega)}}{\|v\|_{\mathcal{D}}} .
$$

A sequence $\left(\mathcal{D}_{m}\right)_{m \in \mathbb{N}}$ of gradient discretisations is said to be coercive if there exists $C_{P} \in \mathbb{R}_{+}$such that $C_{\mathcal{D}_{m}} \leq C_{P}$ for all $m \in \mathbb{N}$.

Remark 2.5 (Discrete Poincaré inequality.) Equation (2.2) yields $\left\|\Pi_{\mathcal{D}} v\right\|_{L^{p}(\Omega)} \leq C_{\mathcal{D}}\left\|\nabla_{\mathcal{D} v}\right\|_{L^{p}(\Omega)^{d}}$.

Consistency is ensured by a proper choice of the reconstruction operator and the discrete gradient.

Definition 2.6 (Consistency) Let $\mathcal{D}$ be a gradient discretisation for Problem (1.1) in the sense of Definition 2.1, and let $S_{\mathcal{D}}: W_{0}^{1, p}(\Omega) \rightarrow[0,+\infty)$ be defined by

$$
\forall \varphi \in W_{0}^{1, p}(\Omega), \quad S_{\mathcal{D}}(\varphi)=\min _{v \in X_{\mathcal{D}, 0}}\left(\left\|\Pi_{\mathcal{D}} v-\varphi\right\|_{L^{p}(\Omega)}+\left\|\nabla_{\mathcal{D}} v-\nabla \varphi\right\|_{L^{p}(\Omega)^{d}}\right)
$$

A sequence $\left(\mathcal{D}_{m}\right)_{m \in \mathbb{N}}$ of gradient discretisations is said to be consistent if, for all $\varphi \in W_{0}^{1, p}(\Omega), S_{\mathcal{D}_{m}}(\varphi)$ tends to 0 as $m \rightarrow \infty$.

Since we are dealing with nonconforming methods, we need to make sure that the dual of the discrete gradient is "close to" a discrete divergence operator. 
Definition 2.7 (Limit-conformity) Let $\mathcal{D}$ be a gradient discretisation for Problem (1.1) in the sense of Definition 2.1. We let $p^{\prime}=\frac{p}{p-1}, W^{\operatorname{div}, p^{\prime}}(\Omega)=\left\{\boldsymbol{\varphi} \in L^{p^{\prime}}(\Omega)^{d}, \operatorname{div} \varphi \in L^{p^{\prime}}(\Omega)\right\}$ and $W_{\mathcal{D}}: W^{\operatorname{div}, p^{\prime}}(\Omega) \rightarrow$ $[0,+\infty)$ be defined by

$$
\forall \boldsymbol{\varphi} \in W^{\operatorname{div}, p^{\prime}}(\Omega), W_{\mathcal{D}}(\boldsymbol{\varphi})=\max _{u \in X_{\mathcal{D}, 0} \backslash\{0\}} \frac{1}{\|u\|_{\mathcal{D}}}\left|\int_{\Omega}\left(\nabla_{\mathcal{D}} u(\boldsymbol{x}) \cdot \boldsymbol{\varphi}(\boldsymbol{x})+\Pi_{\mathcal{D}} u(\boldsymbol{x}) \operatorname{div} \boldsymbol{\varphi}(\boldsymbol{x})\right) \mathrm{d} \boldsymbol{x}\right| .
$$

A sequence $\left(\mathcal{D}_{m}\right)_{m \in \mathbb{N}}$ of gradient discretisations is said to be limit-conforming if, for all $\varphi \in W^{\text {div }, p^{\prime}}(\Omega)$, $W_{\mathcal{D}_{m}}(\varphi)$ tends to 0 as $m \rightarrow \infty$.

Dealing with generic non-linearities often requires compactness properties on the scheme.

Definition 2.8 (Compactness) Let $\mathcal{D}$ be a gradient discretisation for Problem (1.1) in the sense of Definition 2.1, and let $T_{\mathcal{D}}: \mathbb{R}^{d} \rightarrow \mathbb{R}^{+}$be defined by

$$
\forall \boldsymbol{\xi} \in \mathbb{R}^{d}, \quad T_{\mathcal{D}}(\boldsymbol{\xi})=\max _{v \in X_{\mathcal{D}, 0} \backslash\{0\}} \frac{\left\|\Pi_{\mathcal{D}} v(\cdot+\boldsymbol{\xi})-\Pi_{\mathcal{D}} v\right\|_{L^{p}\left(\mathbb{R}^{d}\right)}}{\|v\|_{\mathcal{D}}},
$$

where $\Pi_{\mathcal{D}} v$ has been extended by 0 outside $\Omega$.

A sequence $\left(\mathcal{D}_{m}\right)_{m \in \mathbb{N}}$ of gradient discretisations is said to be compact if the following uniform limit holds:

$$
\lim _{|\boldsymbol{\xi}| \rightarrow 0} \sup _{m \in \mathbb{N}} T_{\mathcal{D}_{m}}(\boldsymbol{\xi})=0
$$

In fact, the consistency and limit-conformity properties of a given gradient scheme only need to be checked on dense subsets of the test functions spaces. The following lemma, useful in Section 5, is an immediate consequence of [21, Lemma 2.4].

Lemma 2.9 (Sufficient conditions) Let $\mathcal{F}$ be a family of gradient discretisations for Problem (1.1) in the sense of Definition 2.1. Assume that there exist $C, \nu \in(0, \infty)$ and, for all $\mathcal{D} \in \mathcal{F}$, a real value $h_{\mathcal{D}} \in(0,+\infty)$ such that:

$$
\begin{aligned}
& C_{\mathcal{D}} \leq C, \\
& S_{\mathcal{D}}(\varphi) \leq C h_{\mathcal{D}}\|\varphi\|_{W^{2, \infty}(\Omega)}, \text { for all } \varphi \in C_{c}^{\infty}(\Omega), \\
& W_{\mathcal{D}}(\varphi) \leq C h_{\mathcal{D}}\|\varphi\|_{\left(W^{1, \infty}\left(\mathbb{R}^{d}\right)\right)^{d}}, \text { for all } \varphi \in C_{c}^{\infty}\left(\mathbb{R}^{d}\right)^{d}, \\
& T_{\mathcal{D}}(\boldsymbol{\xi}) \leq C|\boldsymbol{\xi}|^{\nu}, \text { for all } \boldsymbol{\xi} \in \mathbb{R}^{d},
\end{aligned}
$$

where $C_{\mathcal{D}}, S_{\mathcal{D}}, W_{\mathcal{D}}$ and $T_{\mathcal{D}}$ are defined by $(2.2)-(2.5)$.

Then, any sequence $\left(\mathcal{D}_{m}\right)_{m \in \mathbb{N}} \subset \mathcal{F}$ such that $h_{\mathcal{D}_{m}} \rightarrow 0$ as $m \rightarrow \infty$ is coercive, consistent, limit-conforming and compact.

Remark 2.10 In several cases, $h_{\mathcal{D}}$ stands for the mesh size. This is for instance the case in the analysis of Hybrid Mimetic Mixed schemes in Section 5.

\section{$3 \quad$ Elliptic problems}

\subsection{Error estimate in the linear case}

We recall an error estimate which was obtained in [21] in the linear case. We consider the following problem, corresponding to (1.1) with $p=2$ and $a(\boldsymbol{x}, s, \boldsymbol{\xi})=\Lambda(\boldsymbol{x}) \boldsymbol{\xi}$ :

$$
\begin{aligned}
& -\operatorname{div}(\Lambda(\boldsymbol{x}) \nabla \bar{u})=f \text { in } \Omega, \\
& \bar{u}=0 \text { on } \partial \Omega,
\end{aligned}
$$


with

$\Lambda: \Omega \rightarrow \mathcal{S}_{d}(\mathbb{R})$ measurable s.t. $\Lambda(\boldsymbol{x})$ has eigenvalues in $(\underline{\lambda}, \bar{\lambda}) \subset(0,+\infty)$ for a.e. $\boldsymbol{x} \in \Omega$, $f \in L^{2}(\Omega)$,

$\left(\mathcal{S}_{d}(\mathbb{R})\right.$ is the set of $d \times d$ symmetric matrices). Under these hypotheses, the weak solution of (1.1) is the unique function $\bar{u}$ satisfying:

$$
\left\{\begin{array}{l}
\bar{u} \in H_{0}^{1}(\Omega), \\
\int_{\Omega} \Lambda(\boldsymbol{x}) \nabla \bar{u}(\boldsymbol{x}) \cdot \nabla \bar{v}(\boldsymbol{x}) \mathrm{d} \boldsymbol{x}=\int_{\Omega} f(\boldsymbol{x}) \bar{v}(\boldsymbol{x}) \mathrm{d} \boldsymbol{x} \quad \forall \bar{v} \in H_{0}^{1}(\Omega) .
\end{array}\right.
$$

Problem (3.3) is approximated by Scheme (2.1) with $a(\boldsymbol{x}, s, \boldsymbol{\xi})=\Lambda(\boldsymbol{x}) \boldsymbol{\xi}$. The following lemma, proved in [21], is in the spirit of the results given in [28].

Lemma 3.1 (Control of the approximation error) Under Hypothesis (3.2), let $\bar{u} \in H_{0}^{1}(\Omega)$ be the solution of (3.3) (remark that since $f \in L^{2}(\Omega)$, one has $\Lambda \nabla \bar{u} \in W^{\mathrm{div}, 2}(\Omega)$ ).

Let $\mathcal{D}$ be a gradient discretisation in the sense of Definition 2.1 with $p=2$. Then there exists one and only one $u_{\mathcal{D}} \in X_{\mathcal{D}, 0}$ solution to the gradient scheme (2.1). This solution moreover satisfies the following inequalities:

$$
\begin{aligned}
& \left\|\nabla \bar{u}-\nabla_{\mathcal{D}} u_{\mathcal{D}}\right\|_{L^{2}(\Omega)^{d}} \leq \frac{1}{\underline{\lambda}}\left[W_{\mathcal{D}}(\Lambda \nabla \bar{u})+(\bar{\lambda}+\underline{\lambda}) S_{\mathcal{D}}(\bar{u})\right], \\
& \left\|\bar{u}-\Pi_{\mathcal{D}} u_{\mathcal{D}}\right\|_{L^{2}(\Omega)} \leq \frac{1}{\underline{\lambda}}\left[C_{\mathcal{D}} W_{\mathcal{D}}(\Lambda \nabla \bar{u})+\left(C_{\mathcal{D}} \bar{\lambda}+\underline{\lambda}\right) S_{\mathcal{D}}(\bar{u})\right],
\end{aligned}
$$

where $C_{\mathcal{D}}, S_{\mathcal{D}}$ and $W_{\mathcal{D}}$ are defined by (2.2)-(2.4).

Remark 3.2 As a consequence, if $\left(u_{\mathcal{D}_{m}}\right)_{m \in \mathbb{N}}$ is a sequence of solutions to (2.1) corresponding to a coercive (Definition 2.4) consistent (Definition 2.6) and limit-conforming (Definition 2.7) sequence of gradient discretisations $\left(\mathcal{D}_{m}\right)_{m \in \mathbb{N}}$, then $\Pi_{\mathcal{D}_{m}} u_{\mathcal{D}_{m}} \rightarrow \bar{u}$ in $L^{2}(\Omega)$ and $\nabla_{\mathcal{D}_{m}} u_{\mathcal{D}_{m}} \rightarrow \nabla \bar{u}$ in $L^{2}(\Omega)^{d}$ as $m \rightarrow \infty$.

We notice that, in this linear case, compactness of the sequence of gradient discretisations is not necessary to obtain these convergences (see also Remark 3.8).

Remark 3.3 Note that, under the assumptions of Lemma 2.9 and regularity assumptions on the solution to (3.1), Lemma 3.1 gives an $\mathcal{O}\left(h_{\mathcal{D}}\right)$ convergence rate of gradient schemes for linear problems.

One can in fact prove, for all gradient schemes mentioned in the introduction, that $S_{\mathcal{D}}(\varphi) \leq C h_{\mathcal{D}}\|\varphi\|_{H^{2}(\Omega)}$ for all $\varphi \in H^{2}(\Omega) \cap H_{0}^{1}(\Omega)$ and $W_{\mathcal{D}}(\varphi) \leq C h_{\mathcal{D}}\|\varphi\|_{H^{1}(\Omega)^{d}}$ for all $\varphi \in H^{1}(\Omega)^{d}$ (where $h_{\mathcal{D}}$ measures the scheme precision and, in many cases, stands for the mesh size). Hence, Lemma 3.1 gives $\mathcal{O}\left(h_{\mathcal{D}}\right)$ error estimates for these methods as soon as $\Lambda \in W^{1, \infty}(\Omega)^{d \times d}$ and $\bar{u} \in H^{2}(\Omega) \cap H_{0}^{1}(\Omega)$.

\subsection{Convergence in the nonlinear case}

We now study the convergence of gradient schemes for the more general nonlinear framework of Problem (1.1). The assumptions we consider are:

$$
\boldsymbol{a}: \Omega \times L^{p}(\Omega) \times \mathbb{R}^{d} \rightarrow \mathbb{R}^{d} \text {, with } p \in(1,+\infty) \text {, is a Caratheodory function, }
$$

(i.e. a function such that, for a.e. $\boldsymbol{x} \in \Omega,(u, \boldsymbol{\xi}) \mapsto \boldsymbol{a}(\boldsymbol{x}, u, \boldsymbol{\xi})$ is continuous and, for any $(u, \boldsymbol{\xi}) \in L^{p}(\Omega) \times \mathbb{R}^{d}$, $\boldsymbol{x} \mapsto \boldsymbol{a}(\boldsymbol{x}, u, \boldsymbol{\xi})$ is measurable)

$$
\begin{aligned}
& \exists \underline{a} \in(0,+\infty): \boldsymbol{a}(\boldsymbol{x}, u, \boldsymbol{\xi}) \cdot \boldsymbol{\xi} \geq \underline{a}|\boldsymbol{\xi}|^{p}, \text { for a.e. } \boldsymbol{x} \in \Omega, \forall u \in L^{p}(\Omega), \forall \boldsymbol{\xi} \in \mathbb{R}^{d}, \\
& (\boldsymbol{a}(\boldsymbol{x}, u, \boldsymbol{\xi})-\boldsymbol{a}(\boldsymbol{x}, u, \boldsymbol{\chi})) \cdot(\boldsymbol{\xi}-\boldsymbol{\chi}) \geq 0, \text { for a.e. } \boldsymbol{x} \in \Omega, \forall u \in L^{p}(\Omega), \forall \boldsymbol{\xi}, \boldsymbol{\chi} \in \mathbb{R}^{d}, \\
& \exists \bar{a} \in L^{p^{\prime}}(\Omega), \exists \mu \in(0,+\infty): \\
& \quad|\boldsymbol{a}(\boldsymbol{x}, u, \boldsymbol{\xi})| \leq \bar{a}(\boldsymbol{x})+\mu|\boldsymbol{\xi}|^{p-1}, \text { for a.e. } \boldsymbol{x} \in \Omega, \forall u \in L^{p}(\Omega), \forall \boldsymbol{\xi} \in \mathbb{R}^{d},
\end{aligned}
$$


and

$$
f \in L^{p^{\prime}}(\Omega) \text { where } p^{\prime}=\frac{p}{p-1} .
$$

Remark 3.4 Note that the dependence of $\boldsymbol{a}$ on $u$ is assumed to be nonlocal: $\boldsymbol{a}(\boldsymbol{x}, u, \cdot)$ depends on all the values of $u \in L^{p}(\Omega)$, not only on $u(\boldsymbol{x})$. These assumptions cover for example the case where $\boldsymbol{a}(\boldsymbol{x}, u, \nabla u(\boldsymbol{x}))=\Lambda[u](\boldsymbol{x}) \nabla u(\boldsymbol{x})$ with $\Lambda: L^{p}(\Omega) \rightarrow L^{\infty}\left(\Omega ; \mathcal{S}_{d}(\mathbb{R})\right)$ as in [10, 14, 29].

These assumptions (in particular (3.4a)) do not allow to cover usual local dependencies $\boldsymbol{a}(\boldsymbol{x}, u(\boldsymbol{x}), \nabla u(\boldsymbol{x}))$ as in the non-monotone operators studied in [26]. However, the adaptation of the following results to the local dependency case is quite easy and more classical. See e.g. [15] for an adaptation of the original Leray-Lions method to a numerical scheme (based on the Mixed Finite Volume method) for local nonmonotone operators.

If a function $\boldsymbol{a}$ satisfies (3.4), then the mapping $u \mapsto-\operatorname{div} \boldsymbol{a}(\cdot, u, \nabla u)$ is called a generalised Leray-Lions operator. A classical example is the $p$-Laplacian operator, obtained by setting $\boldsymbol{a}(\boldsymbol{x}, u, \boldsymbol{\xi})=|\boldsymbol{\xi}|^{p-2} \boldsymbol{\xi}$. Note that the existence of at least one solution to (1.1) is shown in [26] under Hypothesis (3.4) in the case where $\boldsymbol{a}$ does not depend on $u$. In our framework, we say that a function $\bar{u}$ is a weak solution to (1.1) if:

$$
\left\{\begin{array}{l}
\bar{u} \in W_{0}^{1, p}(\Omega), \\
\int_{\Omega} \boldsymbol{a}(\boldsymbol{x}, \bar{u}, \nabla \bar{u}(\boldsymbol{x})) \cdot \nabla \bar{v}(\boldsymbol{x}) \mathrm{d} \boldsymbol{x}=\int_{\Omega} f(\boldsymbol{x}) \bar{v}(\boldsymbol{x}) \mathrm{d} \boldsymbol{x}, \quad \forall \bar{v} \in W_{0}^{1, p}(\Omega) .
\end{array}\right.
$$

Remark 3.5 Note that, even if $\boldsymbol{a}$ does not depend on $u \in L^{p}(\Omega)$, the solution to (3.6) is not necessarily unique. Consider the case where $p=2, d=1, \Omega=]-1,2[, f(x)=0$ for $x \in(-1,0) \cup(1,2), f(x)=2$ for $x \in(0,1)$, and

$$
\boldsymbol{a}(\boldsymbol{x}, u, \boldsymbol{\xi})=(\min (|\boldsymbol{\xi}|, 1)+\max (|\boldsymbol{\xi}|-2,0)) \frac{\boldsymbol{\xi}}{|\boldsymbol{\xi}|}, \forall \boldsymbol{\xi} \in \mathbb{R}, \quad \forall u \in L^{2}(\Omega) .
$$

Then (3.4b) is satisfied with $\underline{a}=\frac{1}{2},(3.4 \mathrm{c})$ is satisfied since $\boldsymbol{a}$ is non-decreasing w.r.t. $\boldsymbol{\xi}$ and $(3.4 \mathrm{~d})$ is satisfied with $\bar{a}(\boldsymbol{x})=0$ and $\mu=1$. Then the function $u(x)=\alpha(x+1)$ for $x \in(-1,0), \alpha+x(1-x)$ for $x \in(0,1), \alpha(2-x)$ for $x \in(1,2)$ is solution to (3.6) for any value $\alpha \in[1,2]$.

The hypothesis that $\boldsymbol{a}$ is strictly monotone, which may be expressed as

$$
(\boldsymbol{a}(\boldsymbol{x}, u, \boldsymbol{\xi})-\boldsymbol{a}(\boldsymbol{x}, u, \boldsymbol{\chi})) \cdot(\boldsymbol{\xi}-\boldsymbol{\chi})>0 \text {, for a.e. } \boldsymbol{x} \in \Omega, \forall u \in L^{p}(\Omega), \forall \boldsymbol{\xi}, \boldsymbol{\chi} \in \mathbb{R}^{d} \text { with } \boldsymbol{\xi} \neq \boldsymbol{\chi},
$$

will only be useful to prove the strong convergence of the approximate gradient.

Theorem 3.6 (Convergence of the scheme) Under Assumptions (3.4)-(3.5), let $\left(\mathcal{D}_{m}\right)_{m \in \mathbb{N}}$ be a sequence of gradient discretisations in the sense of Definition 2.1, which is coercive, consistent, limitconforming and compact in the sense of Definitions 2.4, 2.6, 2.7 and 2.8.

Then, for any $m \in \mathbb{N}$, there exists at least one $u_{\mathcal{D}_{m}} \in X_{\mathcal{D}_{m}, 0}$ solution to the gradient scheme (2.1) and, up to a subsequence, $\Pi_{\mathcal{D}_{m}} u_{\mathcal{D}_{m}}$ converges strongly in $L^{p}(\Omega)$ to a solution $\bar{u}$ of (3.6) and $\nabla_{\mathcal{D}_{m}} u_{\mathcal{D}_{m}}$ converges weakly in $L^{p}(\Omega)^{d}$ to $\nabla \bar{u}$ as $m \rightarrow \infty$. Moreover, if we assume that the Leray-Lions operator $\boldsymbol{a}$ is strictly monotone in the sense of (3.7), then $\nabla_{\mathcal{D}_{m}} u_{\mathcal{D}_{m}}$ converges strongly in $L^{p}(\Omega)^{d}$ to $\nabla \bar{u}$ as $m \rightarrow \infty$.

In the case where the solution $\bar{u}$ of (3.6) is unique, then the whole sequence converges to $\bar{u}$ as $m \rightarrow \infty$ in the senses above.

Remark 3.7 As a by-product, this theorem also gives the existence of a solution $\bar{u}$ to (3.6). Indeed, under the assumptions of the theorem, the proof shows that the sequence $u_{\mathcal{D}_{m}}$ has a converging subsequence and that the limit $\bar{u}$ of this subsequence is in fact a solution to the continuous problem. Since there exists at least one gradient scheme which satisfies the assumptions of this theorem (for example, the HMM method - see Section 5), this gives the existence of a solution to (3.6). 
Remark 3.8 In the case where $\boldsymbol{a}$ does not depend on $u \in L^{p}(\Omega)$, the proof of the weak convergence of $\Pi_{\mathcal{D}_{m}} u_{\mathcal{D}_{m}}$ to a solution of (3.6) does not require the compactness of the sequence of gradient discretisations. In this case the strong convergence of $\Pi_{\mathcal{D}_{m}} u_{\mathcal{D}_{m}}$ results from (3.7) (which gives the strong convergence of the approximate gradient) and from the coercivity and the consistency of the sequence $\left(\mathcal{D}_{m}\right)_{m \in \mathbb{N}}$.

\section{Proof}

This proof follows the same ideas as in $[15,19]$.

\section{Step 1: existence of a solution to the scheme}

Let $\mathcal{D}$ be a gradient discretisation in the sense of Definition 2.1. We endow the finite dimensional space $X_{\mathcal{D}, 0}$ with an inner product $\langle$,$\rangle and we denote by |\cdot|$ the norm coming from this inner product. We define $F: X_{\mathcal{D}, 0} \rightarrow X_{\mathcal{D}, 0}$ as the function such that, if $u \in X_{\mathcal{D}, 0}, F(u)$ is the unique element in $X_{\mathcal{D}, 0}$ which satisfies

$$
\forall v \in X_{\mathcal{D}, 0}, \quad\langle F(u), v\rangle=\int_{\Omega} \boldsymbol{a}\left(\boldsymbol{x}, \Pi_{\mathcal{D}} u, \nabla_{\mathcal{D}} u(\boldsymbol{x})\right) \cdot \nabla_{\mathcal{D}} v(\boldsymbol{x}) \mathrm{d} \boldsymbol{x} .
$$

Likewise, we denote by $w \in X_{\mathcal{D}, 0}$ the unique element such that

$$
\forall v \in X_{\mathcal{D}, 0}, \quad\langle w, v\rangle=\int_{\Omega} f(\boldsymbol{x}) \Pi_{\mathcal{D}} v(\boldsymbol{x}) \mathrm{d} \boldsymbol{x} .
$$

The properties of $\boldsymbol{a}$ show that $F$ is continuous and that, for all $u \in X_{\mathcal{D}, 0},\langle F(u), u\rangle \geq \underline{a}\|u\|_{\mathcal{D}}^{p}$. By equivalence of the norms $|\cdot|$ and $\|\cdot\|_{\mathcal{D}}$ on $X_{\mathcal{D}, 0}$, we deduce that $\langle F(u), u\rangle \geq C_{1}|u|^{p}$ with $C_{1}$ not depending on $u$. This shows that $\lim _{|u| \rightarrow \infty} \frac{\langle F(u), u\rangle}{|u|}=+\infty$ and thus that $F$ is surjective (see [26] or [12, Theorem 3.3, page 19]). There exists therefore $u_{\mathcal{D}} \in X_{\mathcal{D}, 0}$ such that $F\left(u_{\mathcal{D}}\right)=w$, and this $u_{\mathcal{D}}$ is a solution to $(2.1)$.

\section{Step 2: convergence to a solution of the continuous problem}

Letting $v=u_{\mathcal{D}_{m}}$ in (2.1) with $\mathcal{D}=\mathcal{D}_{m}$ and using (2.2) and Hypothesis (3.4b), we get

$$
\underline{a}\left\|\nabla_{\mathcal{D}_{m}} u_{\mathcal{D}_{m}}\right\|_{L^{p}(\Omega)^{d}}^{p-1} \leq C_{\mathcal{D}_{m}}\|f\|_{L^{p^{\prime}}(\Omega)} .
$$

Thanks to the coercivity of the sequence of gradient discretisations, this provides an estimate on $\nabla_{\mathcal{D}_{m}} u_{\mathcal{D}_{m}}$ in $L^{p}(\Omega)^{d}$ and on $\Pi_{\mathcal{D}_{m}} u_{\mathcal{D}_{m}}$ in $L^{p}(\Omega)$. By Hypothesis $(3.4 \mathrm{~d})$, the sequence of functions $\mathcal{A}_{\mathcal{D}_{m}}(\boldsymbol{x})=$ $\boldsymbol{a}\left(\boldsymbol{x}, \Pi_{\mathcal{D}_{m}} u_{\mathcal{D}_{m}}, \nabla_{\mathcal{D}_{m}} u_{\mathcal{D}_{m}}(\boldsymbol{x})\right)$ remains bounded in $L^{p^{\prime}}(\Omega)^{d}$. Extending $\Pi_{\mathcal{D}_{m}} u_{\mathcal{D}_{m}}$ and $\nabla_{\mathcal{D}_{m}} u_{\mathcal{D}_{m}}$ by 0 outside $\Omega$, we infer the existence of $\bar{u} \in L^{p}\left(\mathbb{R}^{d}\right), \overline{\boldsymbol{G}} \in L^{p}\left(\mathbb{R}^{d}\right)^{d}$ and $\boldsymbol{A} \in L^{p^{\prime}}(\Omega)^{d}$ such that, up to a subsequence again denoted by $\left(\mathcal{D}_{m}\right)_{m \in \mathbb{N}}, \nabla_{\mathcal{D}_{m}} u_{\mathcal{D}_{m}}$ converges weakly to $\overline{\boldsymbol{G}}$ in $L^{p}\left(\mathbb{R}^{d}\right)^{d}, \Pi_{\mathcal{D}_{m}} u_{\mathcal{D}_{m}}$ converges weakly to $\bar{u}$ in $L^{p}\left(\mathbb{R}^{d}\right)$ and $\mathcal{A}_{\mathcal{D}_{m}}$ converges weakly to $\boldsymbol{A}$ in $L^{p^{\prime}}(\Omega)^{d}$, as $m \rightarrow \infty$.

Thanks to the limit-conformity of the sequence of discretisations, passing to the limit in (2.4) we get that

$$
\int_{\Omega}(\overline{\boldsymbol{G}}(\boldsymbol{x}) \cdot \boldsymbol{\varphi}(\boldsymbol{x})+\bar{u}(\boldsymbol{x}) \operatorname{div} \boldsymbol{\varphi}(\boldsymbol{x})) \mathrm{d} \boldsymbol{x}=0, \forall \boldsymbol{\varphi} \in W^{\operatorname{div}, p^{\prime}}(\Omega) .
$$

Since $\overline{\boldsymbol{G}}=0$ and $\bar{u}=0$ outside $\Omega$, the above relation may be written for all $\varphi \in W^{\text {div, } p^{\prime}}\left(\mathbb{R}^{d}\right)$ with integration on $\mathbb{R}^{d}$, which proves that $\overline{\boldsymbol{G}}=\nabla \bar{u}$ and that the restriction of $\bar{u}$ to $\Omega$, again denoted by $\bar{u}$, belongs to $W_{0}^{1, p}(\Omega)$. Finally, the compactness of the sequence of gradient discretisations and Kolmogorov's theorem give the strong convergence of $\Pi_{\mathcal{D}_{m}} u_{\mathcal{D}_{m}}$ to $\bar{u}$ in $L^{p}\left(\mathbb{R}^{d}\right.$ ) (this strong convergence is only necessary for coping with the dependence upon $u$ of $\boldsymbol{a}$ ).

Let us now show that $\bar{u}$ is solution to (3.6), using the well-known Minty trick [27]. For a given $\varphi \in W_{0}^{1, p}(\Omega)$ and for any gradient discretisation $\mathcal{D}$ belonging to the sequence $\left(\mathcal{D}_{m}\right)_{m \in \mathbb{N}}$, we introduce

$$
P_{\mathcal{D}} \varphi=\underset{v \in X_{\mathcal{D}, 0}}{\operatorname{argmin}}\left(\left\|\Pi_{\mathcal{D}} v-\varphi\right\|_{L^{p}(\Omega)}+\left\|\nabla_{\mathcal{D}} v-\nabla \varphi\right\|_{L^{p}(\Omega)^{d}}\right)
$$


as a test function in (2.1). By the consistency of $\left(\mathcal{D}_{m}\right)_{m \in \mathbb{N}}$, letting $m \rightarrow \infty$ we get

$$
\int_{\Omega} \boldsymbol{A}(\boldsymbol{x}) \cdot \nabla \varphi(\boldsymbol{x}) \mathrm{d} \boldsymbol{x}=\int_{\Omega} f(\boldsymbol{x}) \varphi(\boldsymbol{x}) \mathrm{d} \boldsymbol{x}, \quad \forall \varphi \in W_{0}^{1, p}(\Omega) .
$$

On the other hand, we may let $m \rightarrow \infty$ in (2.1) with $u_{\mathcal{D}_{m}}$ as a test function. Using (3.8) with $\varphi=\bar{u}$, this leads to

$$
\lim _{m \rightarrow \infty} \int_{\Omega} \boldsymbol{a}\left(\boldsymbol{x}, \Pi_{\mathcal{D}_{m}} u_{\mathcal{D}_{m}}, \nabla_{\mathcal{D}_{m}} u_{\mathcal{D}_{m}}(\boldsymbol{x})\right) \cdot \nabla_{\mathcal{D}_{m}} u_{\mathcal{D}_{m}}(\boldsymbol{x}) \mathrm{d} \boldsymbol{x}=\int_{\Omega} f(\boldsymbol{x}) \bar{u}(\boldsymbol{x}) \mathrm{d} \boldsymbol{x}=\int_{\Omega} \boldsymbol{A}(\boldsymbol{x}) \cdot \nabla \bar{u}(\boldsymbol{x}) \mathrm{d} \boldsymbol{x} .
$$

Hypothesis (3.4c) gives, for any $\boldsymbol{G} \in L^{p}(\Omega)^{d}$,

$$
\int_{\Omega}\left(\boldsymbol{a}\left(\boldsymbol{x}, \Pi_{\mathcal{D}_{m}} u_{\mathcal{D}_{m}}, \nabla_{\mathcal{D}_{m}} u_{\mathcal{D}_{m}}(\boldsymbol{x})\right)-\boldsymbol{a}\left(\boldsymbol{x}, \Pi_{\mathcal{D}_{m}} u_{\mathcal{D}_{m}}, \boldsymbol{G}(\boldsymbol{x})\right)\right) \cdot\left(\nabla_{\mathcal{D}_{m}} u_{\mathcal{D}_{m}}(\boldsymbol{x})-\boldsymbol{G}(\boldsymbol{x})\right) \mathrm{d} \boldsymbol{x} \geq 0 .
$$

Developing this inequality and using (3.9) for the one term involving a product of two weak convergences, we may let $m \rightarrow \infty$ and we get

$$
\int_{\Omega}(\boldsymbol{A}(\boldsymbol{x})-\boldsymbol{a}(\boldsymbol{x}, \bar{u}, \boldsymbol{G}(\boldsymbol{x}))) \cdot(\nabla \bar{u}(\boldsymbol{x})-\boldsymbol{G}(\boldsymbol{x})) \mathrm{d} \boldsymbol{x} \geq 0, \forall \boldsymbol{G} \in L^{p}(\Omega)^{d} .
$$

We then set $\boldsymbol{G}=\nabla \bar{u}+\alpha \boldsymbol{\varphi}$ in the preceding inequality, where $\boldsymbol{\varphi} \in C_{c}^{\infty}(\Omega)^{d}$ and $\alpha>0$. Dividing by $\alpha$, we get

$$
-\int_{\Omega}(\boldsymbol{A}(\boldsymbol{x})-\boldsymbol{a}(\boldsymbol{x}, \bar{u}, \nabla \bar{u}(\boldsymbol{x})+\alpha \boldsymbol{\varphi}(\boldsymbol{x}))) \cdot \boldsymbol{\varphi}(\boldsymbol{x}) \mathrm{d} \boldsymbol{x} \geq 0, \forall \boldsymbol{\varphi} \in C_{c}^{\infty}(\Omega)^{d}, \forall \alpha>0 .
$$

We then let $\alpha \rightarrow 0$ and use the dominated convergence theorem, which leads to

$$
-\int_{\Omega}(\boldsymbol{A}(\boldsymbol{x})-\boldsymbol{a}(\boldsymbol{x}, \bar{u}, \nabla \bar{u}(\boldsymbol{x}))) \cdot \boldsymbol{\varphi}(\boldsymbol{x}) \mathrm{d} \boldsymbol{x} \geq 0, \forall \boldsymbol{\varphi} \in C_{c}^{\infty}(\Omega)^{d} .
$$

Changing $\varphi$ into $-\varphi$, we deduce that

$$
\int_{\Omega}(\boldsymbol{A}(\boldsymbol{x})-\boldsymbol{a}(\boldsymbol{x}, \bar{u}, \nabla \bar{u}(\boldsymbol{x}))) \cdot \boldsymbol{\varphi}(\boldsymbol{x}) \mathrm{d} \boldsymbol{x}=0, \forall \boldsymbol{\varphi} \in C_{c}^{\infty}(\Omega)^{d},
$$

and therefore that

$$
\boldsymbol{A}(\boldsymbol{x})=\boldsymbol{a}(\boldsymbol{x}, \bar{u}, \nabla \bar{u}(\boldsymbol{x})) \text {, for a.e. } \boldsymbol{x} \in \Omega .
$$

In addition to (3.8), this shows that $\bar{u}$ is a solution to (3.6). This concludes the proof of the convergence of $\Pi_{\mathcal{D}_{m}} u_{\mathcal{D}_{m}}$ to $\bar{u}$ in $L^{p}(\Omega)$ and of $\nabla_{\mathcal{D}_{m}} u_{\mathcal{D}_{m}}$ to $\nabla \bar{u}$ weakly in $L^{p}(\Omega)^{d}$ as $m \rightarrow \infty$.

\section{Step 3: Assuming now hypothesis (3.7), strong convergence of the approximate gradient}

We follow here the ideas of [26]. Thanks to (3.9) and (3.10), we get

$$
\lim _{m \rightarrow \infty} \int_{\Omega}\left(\boldsymbol{a}\left(\boldsymbol{x}, \Pi_{\mathcal{D}_{m}} u_{\mathcal{D}_{m}}, \nabla_{\mathcal{D}_{m}} u_{\mathcal{D}_{m}}(\boldsymbol{x})\right)-\boldsymbol{a}\left(\boldsymbol{x}, \Pi_{\mathcal{D}_{m}} u_{\mathcal{D}_{m}}, \nabla \bar{u}(\boldsymbol{x})\right)\right) \cdot\left(\nabla_{\mathcal{D}_{m}} u_{\mathcal{D}_{m}}(\boldsymbol{x})-\nabla \bar{u}(\boldsymbol{x})\right) \mathrm{d} \boldsymbol{x}=0 .
$$

Since $\left(\boldsymbol{a}\left(\boldsymbol{x}, \Pi_{\mathcal{D}_{m}} u_{\mathcal{D}_{m}}, \nabla_{\mathcal{D}_{m}} u_{\mathcal{D}_{m}}\right)-\boldsymbol{a}\left(\boldsymbol{x}, \Pi_{\mathcal{D}_{m}} u_{\mathcal{D}_{m}}, \nabla \bar{u}\right)\right) \cdot\left(\nabla_{\mathcal{D}_{m}} u_{\mathcal{D}_{m}}-\nabla \bar{u}\right) \geq 0$ for a.e. $\boldsymbol{x} \in \Omega$, we then have

$$
\left(\boldsymbol{a}\left(\cdot, \Pi_{\mathcal{D}_{m}} u_{\mathcal{D}_{m}}, \nabla_{\mathcal{D}_{m}} u_{\mathcal{D}_{m}}\right)-\boldsymbol{a}\left(\cdot, \Pi_{\mathcal{D}_{m}} u_{\mathcal{D}_{m}}, \nabla \bar{u}\right)\right) \cdot\left(\nabla_{\mathcal{D}_{m}} u_{\mathcal{D}_{m}}-\nabla \bar{u}\right) \rightarrow 0 \text { in } L^{1}(\Omega),
$$

and therefore a.e. for a sub-sequence. Then, thanks to the strict monotony assumption (3.7), we may use Lemma 3.9 given below to show that $\nabla_{\mathcal{D}_{m}} u_{\mathcal{D}_{m}} \rightarrow \nabla \bar{u}$ a.e. as $m \rightarrow \infty$, at least for the same sub-sequence. This shows the a.e. convergence of $\boldsymbol{a}\left(\cdot, \Pi_{\mathcal{D}_{m}} u_{\mathcal{D}_{m}}, \nabla_{\mathcal{D}_{m}} u_{\mathcal{D}_{m}}\right) \cdot \nabla_{\mathcal{D}} u_{\mathcal{D}}$ to $\boldsymbol{a}(\cdot, \bar{u}, \nabla \bar{u}) \cdot \nabla \bar{u}$. We next recall that, by (3.9) and (3.10),

$$
\lim _{m \rightarrow \infty} \int_{\Omega} \boldsymbol{a}\left(\boldsymbol{x}, \Pi_{\mathcal{D}_{m}} u_{\mathcal{D}_{m}}, \nabla_{\mathcal{D}_{m}} u_{\mathcal{D}_{m}}(\boldsymbol{x})\right) \cdot \nabla_{\mathcal{D}_{m}} u_{\mathcal{D}_{m}}(\boldsymbol{x}) d \boldsymbol{x}=\int_{\Omega} \boldsymbol{a}(\boldsymbol{x}, \bar{u}, \nabla \bar{u}(\boldsymbol{x})) \cdot \nabla \bar{u}(\boldsymbol{x}) d \boldsymbol{x} .
$$


Since $\boldsymbol{a}\left(\cdot, \Pi_{\mathcal{D}_{m}} u_{\mathcal{D}_{m}}, \nabla_{\mathcal{D}_{m}} u_{\mathcal{D}_{m}}\right) \cdot \nabla_{\mathcal{D}_{m}} u_{\mathcal{D}_{m}} \geq 0$, we can apply Lemma 3.10 to get $\boldsymbol{a}\left(\cdot, \Pi_{\mathcal{D}_{m}} u_{\mathcal{D}_{m}}, \nabla_{\mathcal{D}_{m}} u_{\mathcal{D}_{m}}\right)$. $\nabla_{\mathcal{D}_{m}} u_{\mathcal{D}_{m}} \rightarrow \boldsymbol{a}(\cdot, \bar{u}, \nabla \bar{u}) \cdot \nabla \bar{u}$ in $L^{1}(\Omega)$ as $m \rightarrow \infty$. This $L^{1}$-convergence gives the equi-integrability of the sequence of functions $\boldsymbol{a}\left(\cdot, \Pi_{\mathcal{D}_{m}} u_{\mathcal{D}_{m}}, \nabla_{\mathcal{D}_{m}} u_{\mathcal{D}_{m}}\right) \cdot \nabla_{\mathcal{D}_{m}} u_{\mathcal{D}_{m}}$, which gives in turn, thanks to (3.4b), the equi-integrability of $\left(\left|\nabla_{\mathcal{D}_{m}} u_{\mathcal{D}_{m}}\right|^{p}\right)_{m \in \mathbb{N}}$. The strong convergence of $\nabla_{\mathcal{D}_{m}} u_{\mathcal{D}_{m}}$ to $\nabla \bar{u}$ in $L^{p}(\Omega)^{d}$ is then a consequence of Vitali's theorem.

Lemma 3.9 Let $B$ be a metric space, let $\boldsymbol{b}$ be a continuous function from $B \times \mathbb{R}^{d}$ to $\mathbb{R}^{d}$ such that

$$
(\boldsymbol{b}(u, \delta)-\boldsymbol{b}(u, \gamma)) \cdot(\delta-\gamma)>0, \forall \delta \neq \gamma \in \mathbb{R}^{d}, \forall u \in B
$$

Let $\left(u_{m}, \beta_{m}\right)_{n \in \mathbb{N}}$ be a sequence in $B \times \mathbb{R}^{d}$ and $(u, \beta) \in B \times \mathbb{R}^{d}$ such that $\left(\boldsymbol{b}\left(u_{m}, \beta_{m}\right)-\boldsymbol{b}\left(u_{m}, \beta\right)\right) \cdot\left(\beta_{m}-\beta\right) \rightarrow 0$ and $u_{m} \rightarrow u$ as $m \rightarrow \infty$. Then $\beta_{m} \rightarrow \beta$ as $m \rightarrow \infty$.

Proof We begin the proof with a preliminary remark. Let $\delta \in \mathbb{R}^{d} \backslash\{0\}$. We define, for all $m \in \mathbb{N}$, the function $h_{\delta, m}: \mathbb{R} \rightarrow \mathbb{R}$ by $h_{\delta, m}(s)=\left(\boldsymbol{b}\left(u_{m}, \beta+s \delta\right)-\boldsymbol{b}\left(u_{m}, \beta\right)\right) \cdot \delta$. The hypothesis on $\boldsymbol{b}$ shows that $h_{\delta, m}$ is an increasing function since, for $s>s^{\prime}$, one has :

$$
h_{\delta, m}(s)-h_{\delta, m}\left(s^{\prime}\right)=\left(\boldsymbol{b}\left(u_{m}, \beta+s \delta\right)-\boldsymbol{b}\left(u_{m}, \beta+s^{\prime} \delta\right)\right) \cdot \delta>0 .
$$

We prove now, by contradiction, that $\lim _{m \rightarrow \infty} \beta_{m}=\beta$. If the sequence $\left(\beta_{m}\right)_{m \in \mathbb{N}}$ does not converge to $\beta$, there exists $\varepsilon>0$ and a subsequence, still denoted by $\left(\beta_{m}\right)_{m \in \mathbb{N}}$, such that $s_{m}:=\left|\beta_{m}-\beta\right| \geq \varepsilon$ for all $m \in \mathbb{N}$. Setting $\delta_{m}=\frac{\beta_{m}-\beta}{\left|\beta_{m}-\beta\right|}$ we can assume, up to a subsequence, that $\delta_{m} \rightarrow \delta$ as $m \rightarrow \infty$, for some $\delta \in \mathbb{R}^{d}$ with $|\delta|=1$. We then have, since $s_{m} \geq \varepsilon$,

$$
\left(\boldsymbol{b}\left(u_{m}, \beta_{m}\right)-\boldsymbol{b}\left(u_{m}, \beta\right)\right) \cdot \frac{\beta_{m}-\beta}{s_{m}}=h_{\delta_{m}, m}\left(s_{m}\right) \geq h_{\delta_{m}, m}(\varepsilon)=\left(\boldsymbol{b}\left(u_{m}, \beta+\varepsilon \delta_{m}\right)-\boldsymbol{b}\left(u_{m}, \beta\right)\right) \cdot \delta_{m} .
$$

Passing to the limit as $m \rightarrow \infty$, we obtain

$$
0=\lim _{m \rightarrow \infty} \frac{1}{s_{m}}\left(\boldsymbol{b}\left(u_{m}, \beta_{m}\right)-\boldsymbol{b}\left(u_{m}, \beta\right)\right) \cdot\left(\beta_{m}-\beta\right) \geq(\boldsymbol{b}(u, \beta+\varepsilon \delta)-\boldsymbol{b}(u, \beta)) \cdot \delta>0,
$$

which is impossible.

The following result is classical (see dro-06-ll,eym-09-cel). Its proof is given for the sake of completeness.

Lemma 3.10 Let $\left(F_{m}\right)_{m \in \mathbb{N}}$ be a sequence non-negative functions in $L^{1}(\Omega)$. Let $F \in L^{1}(\Omega)$ be such that $F_{m} \rightarrow F$ a.e. in $\Omega$ and $\int_{\Omega} F_{m}(\boldsymbol{x}) d \boldsymbol{x} \rightarrow \int_{\Omega} F(\boldsymbol{x}) d \boldsymbol{x}$, as $m \rightarrow \infty$. Then $F_{m} \rightarrow F$ in $L^{1}(\Omega)$ as $m \rightarrow \infty$.

Proof Applying the Dominated Convergence Theorem to the sequence $\left(F-F_{m}\right)^{+}$leads to $\int_{\Omega}(F(\boldsymbol{x})-$ $\left.F_{m}(\boldsymbol{x})\right)^{+} d \boldsymbol{x} \rightarrow 0$ as $m \rightarrow \infty$. Then, since $\left|F-F_{m}\right|=2\left(F-F_{m}\right)^{+}-\left(F-F_{m}\right)$, we conclude that $F_{m} \rightarrow F$ in $L^{1}(\Omega)$ as $m \rightarrow \infty$.

\section{Evolution problems}

In this section, we consider the evolution problem (1.2) under Hypotheses (3.4) and

$$
\begin{aligned}
& T \in(0,+\infty), \\
& u_{\text {ini }} \in L^{2}(\Omega), \\
& f \in L^{p^{\prime}}(\Omega \times(0, T)) \text { where } p^{\prime}=\frac{p}{p-1} .
\end{aligned}
$$

The precise notion of solution to (1.2) that we consider is the following:

$$
\left\{\begin{array}{l}
\bar{u} \in L^{p}\left(0, T ; W_{0}^{1, p}(\Omega)\right) \cap C^{0}\left([0, T] ; L^{2}(\Omega)\right), \partial_{t} \bar{u} \in L^{p^{\prime}}\left(0, T ; W^{-1, p^{\prime}}(\Omega)\right), \\
\bar{u}(\cdot, 0)=u_{\mathrm{ini}} \\
\int_{0}^{T}\left\langle\partial_{t} \bar{u}(\cdot, t), \bar{v}(\cdot, t)\right\rangle_{W^{-1, p^{\prime}}(\Omega), W_{0}^{1, p}(\Omega)} \mathrm{d} t+\int_{0}^{T} \int_{\Omega} \boldsymbol{a}(\boldsymbol{x}, \bar{u}(\cdot, t), \nabla \bar{u}(\boldsymbol{x}, t)) \cdot \nabla \bar{v}(\boldsymbol{x}, t) \mathrm{d} \boldsymbol{x} \mathrm{d} t \\
\quad=\int_{0}^{T} \int_{\Omega} f(\boldsymbol{x}, t) \bar{v}(\boldsymbol{x}, t) \mathrm{d} \boldsymbol{x} \mathrm{d} t, \quad \forall \bar{v} \in L^{p}\left(0 ; T ; W_{0}^{1, p}(\Omega)\right) .
\end{array}\right.
$$


Remark 4.1 The derivative $\partial_{t} \bar{u}$ is to be understood in the usual sense of distributions on $\Omega \times(0, T)$. Since the set $\mathcal{T}=\left\{\sum_{i=1}^{q} \varphi_{i}(t) \gamma_{i}(\boldsymbol{x}): q \in \mathbb{N}, \varphi_{i} \in C_{c}^{\infty}(0, T), \gamma_{i} \in C_{c}^{\infty}(\Omega)\right\}$ of tensorial functions in $C^{\infty}(\Omega \times(0, T))$ is dense in $L^{p}\left(0, T ; W_{0}^{1, p}(\Omega)\right)$, one can ensure that this distribution derivative $\partial_{t} \bar{u}$ belongs to $L^{p^{\prime}}\left(0, T ; W^{-1, p^{\prime}}(\Omega)\right)=\left(L^{p}\left(0, T ; W_{0}^{1, p}(\Omega)\right)^{\prime}\right.$ by checking that the linear form

$$
\varphi \in \mathcal{T} \mapsto\left\langle\partial_{t} \bar{u}, \varphi\right\rangle_{\mathcal{D}^{\prime}, \mathcal{D}}=-\int_{0}^{T} \int_{\Omega} \bar{u}(\boldsymbol{x}, t) \partial_{t} \varphi(\boldsymbol{x}, t) \mathrm{d} \boldsymbol{x} \mathrm{d} t
$$

is continuous for the norm of $L^{p}\left(0, T ; W_{0}^{1, p}(\Omega)\right)$.

Definition 4.2 (Space-time gradient discretisation) Let $p \in(1,+\infty)$ let $\Omega$ be an open subset of $\mathbb{R}^{d}$, with $d \in \mathbb{N}^{\star}$ and let $T>0$ be given. We say that $\mathcal{D}=\left(X_{\mathcal{D}, 0}, \Pi_{\mathcal{D}}, \nabla_{\mathcal{D}},\left(t^{(n)}\right)_{n=0, \ldots, N}\right)$ is a space-time gradient discretisation if

- $\left(X_{\mathcal{D}, 0}, \Pi_{\mathcal{D}}, \nabla_{\mathcal{D}}\right)$ is a gradient discretisation of $\Omega$, in the sense of Definition 2.1, which satisfies $\Pi_{\mathcal{D}}\left(X_{\mathcal{D}, 0}\right) \subset L^{\max (p, 2)}(\Omega)$,

- $t^{(0)}=0<t^{(1)} \ldots<t^{(N)}=T$.

We then set $\delta t^{\left(n+\frac{1}{2}\right)}=t^{(n+1)}-t^{(n)}$, for $n=0, \ldots, N-1$, and $\delta t_{\mathcal{D}}=\max _{n=0, \ldots, N-1} \delta t^{\left(n+\frac{1}{2}\right)}$.

Let $\mathcal{D}=\left(X_{\mathcal{D}, 0}, \Pi_{\mathcal{D}}, \nabla_{\mathcal{D}},\left(t^{(n)}\right)_{n=0, \ldots, N}\right)$ be a space-time gradient discretisation in the sense of Definition 4.2. We define, for a given $\alpha \in\left[\frac{1}{2}, 1\right]$, the following scheme for the discretisation of Problem (1.2): we take $u^{(0)} \in X_{\mathcal{D}, 0}$ and consider a sequence $\left(u^{(n)}\right)_{n=0, \ldots, N} \subset X_{\mathcal{D}, 0}$ such that, for all $n=0, \ldots, N-1$,

$$
\left\{\begin{array}{l}
\text { Setting } u^{(n+\alpha)}=\alpha u^{(n+1)}+(1-\alpha) u^{(n)} \text { and } \delta_{\mathcal{D}}^{\left(n+\frac{1}{2}\right)} u=\frac{u^{(n+1)}-u^{(n)}}{\delta t^{\left(n+\frac{1}{2}\right)}}, \text { we have: } \\
\int_{\Omega}\left[\Pi_{\mathcal{D}} \delta_{\mathcal{D}}^{\left(n+\frac{1}{2}\right)} u(\boldsymbol{x}) \Pi_{\mathcal{D}} v(\boldsymbol{x})+\boldsymbol{a}\left(\boldsymbol{x}, \Pi_{\mathcal{D}} u^{(n+\alpha)}, \nabla_{\mathcal{D}} u^{(n+\alpha)}(\boldsymbol{x})\right) \cdot \nabla_{\mathcal{D}} v(\boldsymbol{x})\right] \mathrm{d} \boldsymbol{x} \\
=\frac{1}{\delta t^{\left(n+\frac{1}{2}\right)}} \int_{t^{(n)}}^{t^{(n+1)}} \int_{\Omega} f(\boldsymbol{x}, t) \Pi_{\mathcal{D}} v(\boldsymbol{x}) \mathrm{d} \boldsymbol{x} \mathrm{d} t, \quad \forall v \in X_{\mathcal{D}, 0} .
\end{array}\right.
$$

Note that the choice $\alpha \geq \frac{1}{2}$ is required for stability reasons and that the choice $\alpha=1$ leads to the implicit scheme. We use the notations $\Pi_{\mathcal{D}}$ and $\nabla_{\mathcal{D}}$ for the definition of space-time dependent functions and we define

$$
\begin{aligned}
& \text { for a.e. } \quad \begin{aligned}
(\boldsymbol{x}, t) & \in \Omega \times\left(t^{(n)}, t^{(n+1)}\right), \forall n=0, \ldots, N-1: \\
\Pi_{\mathcal{D}}^{(\nu)} u(\boldsymbol{x}, t) & =\Pi_{\mathcal{D}} u^{(n+\nu)}(\boldsymbol{x})(\text { for } \nu=\alpha \text { or } 1), \nabla_{\mathcal{D}} u(\boldsymbol{x}, t)=\nabla_{\mathcal{D}} u^{(n+\alpha)}(\boldsymbol{x}), \delta_{\mathcal{D}} u(t)=\delta_{\mathcal{D}}^{\left(n+\frac{1}{2}\right)} u .
\end{aligned}
\end{aligned}
$$

Lemma $4.3\left(L^{\infty}\left(0, T ; L^{2}(\Omega)\right)\right.$ estimate, discrete $L^{p}\left(0, T ; W_{0}^{1, p}(\Omega)\right)$ estimate and existence of a discrete solution)

Under Hypotheses (3.4) and (4.1), let $\mathcal{D}$ be a space-time gradient discretisation in the sense of Definition 4.2. Then there exists at least one solution to Scheme (4.3) and there exists $C_{2}>0$, only depending on $p, C_{P} \geq C_{\mathcal{D}}, C_{\mathrm{ini}} \geq\left\|u_{\mathrm{ini}}-\Pi_{\mathcal{D}} u^{(0)}\right\|_{L^{2}(\Omega)}, u_{\mathrm{ini}}, f, \underline{a}$ such that, for any solution $u$ to this scheme,

$$
\left\|\Pi_{\mathcal{D}}^{(1)} u\right\|_{L^{\infty}\left(0, T ; L^{2}(\Omega)\right)} \leq C_{2},\left\|\Pi_{\mathcal{D}}^{(\alpha)} u\right\|_{L^{\infty}\left(0, T ; L^{2}(\Omega)\right)} \leq C_{2} \text { and }\left\|\nabla_{\mathcal{D}} u\right\|_{L^{p}(\Omega \times(0, T))^{d}} \leq C_{2} .
$$

Proof Let us first prove the estimates. We let $v=\delta t^{\left(n+\frac{1}{2}\right)} u^{(n+\alpha)}$ in (4.3). Since

$$
\delta t^{\left(n+\frac{1}{2}\right)} \Pi_{\mathcal{D}} \delta_{\mathcal{D}}^{\left(n+\frac{1}{2}\right)} u \Pi_{\mathcal{D}} u^{(n+\alpha)}=\frac{1}{2}\left(\left(\Pi_{\mathcal{D}} u^{(n+1)}\right)^{2}-\left(\Pi_{\mathcal{D}} u^{(n)}\right)^{2}\right)+\left(\alpha-\frac{1}{2}\right)\left(\Pi_{\mathcal{D}} u^{(n+1)}-\Pi_{\mathcal{D}} u^{(n)}\right)^{2},
$$

we get, by summing on $n=0, \ldots, m-1$ for a given $m=1, \ldots, N$,

$$
\begin{aligned}
\frac{1}{2}\left\|\Pi_{\mathcal{D}} u^{(m)}\right\|_{L^{2}(\Omega)}^{2}+\underline{a} & \int_{0}^{t^{(m)}}\left\|\nabla_{\mathcal{D}} u(\cdot, t)\right\|_{L^{p}(\Omega)^{d}}^{p} \mathrm{~d} t \\
& \leq\|f\|_{L^{p^{\prime}\left(\Omega \times\left(0, t^{(m)}\right)\right)}}\left\|\Pi_{\mathcal{D}}^{(\alpha)} u\right\|_{L^{p}\left(\Omega \times\left(0, t^{(m)}\right)\right)}+\frac{1}{2}\left\|\Pi_{\mathcal{D}} u^{(0)}\right\|_{L^{2}(\Omega)}^{2} .
\end{aligned}
$$


This leads, thanks to the Young inequality, to

$$
\begin{aligned}
\frac{1}{2}\left\|\Pi_{\mathcal{D}} u^{(m)}\right\|_{L^{2}(\Omega)}^{2} & +\underline{a} \int_{0}^{t^{(m)}}\left\|\nabla_{\mathcal{D}} u(\cdot, t)\right\|_{L^{p}(\Omega)^{d}}^{p} \mathrm{~d} t \\
& \leq \frac{2^{1 /(p-1)} C_{\mathcal{D}}^{p^{\prime}}}{(p \underline{a})^{1 /(p-1)} p^{\prime}}\|f\|_{L^{p^{\prime}\left(\Omega \times\left(0, t^{(m)}\right)\right)}}^{p^{\prime}}+\frac{\underline{a}}{2 C_{\mathcal{D}}^{p}}\left\|\Pi_{\mathcal{D}}^{(\alpha)} u\right\|_{L^{p}\left(\Omega \times\left(0, t^{(m)}\right)\right)}^{p}+\frac{1}{2}\left\|\Pi_{\mathcal{D}} u^{(0)}\right\|_{L^{2}(\Omega)}^{2}
\end{aligned}
$$

Applying (2.2) proves the estimates on $\Pi_{\mathcal{D}}^{(1)} u$ and $\nabla_{\mathcal{D}} u$. The estimate on $\Pi_{\mathcal{D}}^{(\alpha)} u$ follows from the inequality $\left\|\Pi_{\mathcal{D}} u^{(n+\alpha)}\right\|_{L^{2}(\Omega)} \leq \alpha\left\|\Pi_{\mathcal{D}} u^{(n+1)}\right\|_{L^{2}(\Omega)}+(1-\alpha)\left\|\Pi_{\mathcal{D}} u^{(n)}\right\|_{L^{2}(\Omega)}$.

The existence for each $n=0, \ldots, N-1$ of at least one solution to (4.3) follows the same proof as that of Theorem 3.6, reasoning on $u^{(n+\alpha)}$ rather than $u^{(n+1)}$ and using the above estimates.

The following semi-norm on $X_{\mathcal{D}, 0}$ will be useful to apply Theorem 5.13 in the appendix.

Definition 4.4 (Dual semi-norm) Under Hypotheses $(3.4)$, let $\mathcal{D}=\left(X_{\mathcal{D}, 0}, \Pi_{\mathcal{D}}, \nabla_{\mathcal{D}}\right)$ be a gradient discretisation of $\Omega$ in the sense of Definition 2.1. We define the following dual semi-norm on $X_{\mathcal{D}, 0}$ :

$$
\forall w \in X_{\mathcal{D}, 0},|w|_{\star, \mathcal{D}}=\sup \left\{\int_{\Omega} \Pi_{\mathcal{D}} w(\boldsymbol{x}) \Pi_{\mathcal{D}} v(\boldsymbol{x}) \mathrm{d} \boldsymbol{x}: v \in X_{\mathcal{D}, 0},\|v\|_{\mathcal{D}}=1\right\} .
$$

\section{Lemma 4.5 (Estimate on the dual semi-norm of the discrete time derivative)}

Under Hypotheses (3.4) and (4.1), let $\mathcal{D}$ be a space-time gradient discretisation in the sense of Definition 4.2. Let $u$ be a solution to Scheme (4.3). Then there exists $C_{3}$, only depending on $p, \mu, \bar{a}, \underline{a}, C_{\mathrm{ini}} \geq$ $\left\|u_{\text {ini }}-\Pi_{\mathcal{D}} u^{(0)}\right\|_{L^{2}(\Omega)}, u_{\text {ini }}, f, T$ and $C_{P} \geq C_{\mathcal{D}}$, such that

$$
\int_{0}^{T}\left|\delta_{\mathcal{D}} u(t)\right|_{\star, \mathcal{D}}^{p^{\prime}} \mathrm{d} t \leq C_{3} .
$$

Proof Let us take $v \in X_{\mathcal{D}, 0}$ as test function in Scheme (4.3). We have, thanks to Assumption (3.4d) on $\boldsymbol{a}$,

$$
\begin{aligned}
\int_{\Omega} \Pi_{\mathcal{D}} \delta_{\mathcal{D}}^{\left(n+\frac{1}{2}\right)} u(\boldsymbol{x}) \Pi_{\mathcal{D}} v(\boldsymbol{x}) \mathrm{d} \boldsymbol{x} \leq \int_{\Omega}\left(\bar{a}(\boldsymbol{x})+\mu\left|\nabla_{\mathcal{D}} u^{(n+\alpha)}(\boldsymbol{x})\right|^{p-1}\right)\left|\nabla_{\mathcal{D}} v(\boldsymbol{x})\right| \mathrm{d} \boldsymbol{x} & \\
& +\frac{1}{\delta t^{\left(n+\frac{1}{2}\right)}} \int_{t^{(n)}}^{t^{(n+1)}} \int_{\Omega} f(\boldsymbol{x}, t) \Pi_{\mathcal{D}} v(\boldsymbol{x}) \mathrm{d} \boldsymbol{x} \mathrm{d} t
\end{aligned}
$$

which leads, thanks to $(2.2)$, to the existence of $C_{4}>0$ only depending on $p, \mu$ such that

$$
\begin{aligned}
& \int_{\Omega} \Pi_{\mathcal{D}} \delta_{\mathcal{D}}^{\left(n+\frac{1}{2}\right)} u(\boldsymbol{x}) \Pi_{\mathcal{D}} v(\boldsymbol{x}) \mathrm{d} \boldsymbol{x} \\
& \quad \leq C_{4}\left(\|\bar{a}\|_{L^{p^{\prime}(\Omega)}}^{p^{\prime}}+\left\|\nabla_{\mathcal{D}} u^{(n+\alpha)}\right\|_{L^{p}(\Omega)^{d}}^{p}+\frac{C_{\mathcal{D}}}{\delta t^{\left(n+\frac{1}{2}\right)}} \int_{t^{(n)}}^{t^{(n+1)}}\|f(\cdot, t)\|_{L^{p^{\prime}}(\Omega)}^{p^{\prime}} \mathrm{d} t\right)^{(p-1) / p}\left\|\nabla_{\mathcal{D}} v\right\|_{L^{p}(\Omega)^{d}}
\end{aligned}
$$

Taking the supremum on $v \in X_{\mathcal{D}, 0}$ such that $\left\|\nabla_{\mathcal{D}} v\right\|_{L^{p}(\Omega)^{d}}=1$ gives an estimate on $\left|\delta_{\mathcal{D}}^{\left(n+\frac{1}{2}\right)} u\right|_{\star, \mathcal{D}}$. The proof is concluded by raising this estimate to the power $p^{\prime}$, multiplying by $\delta t^{\left(n+\frac{1}{2}\right)}$, summing on $n$ and estimating $\left\|\nabla_{\mathcal{D}} u\right\|_{L^{p}(\Omega \times(0, T))^{d}}^{p}$ thanks to Lemma 4.3.

In order to prove the convergence of the scheme, we shall use the assumptions of coercivity, limitconformity and compactness already used for steady state problems. However, in order to pass to the limit on the time term, we need a modified consistency property for the sequence of gradient discretisations. 
Definition 4.6 (Space-time consistency) Let $\mathcal{D}$ be a space-time gradient discretisation for Problem (1.2) in the sense of Definition 4.2 and let $\widehat{S}_{\mathcal{D}}: W_{0}^{1, p}(\Omega) \cap L^{2}(\Omega) \rightarrow[0,+\infty)$ be defined by

$$
\forall \varphi \in W_{0}^{1, p}(\Omega), \quad \widehat{S}_{\mathcal{D}}(\varphi)=\min _{v \in X_{\mathcal{D}, 0}}\left(\left\|\Pi_{\mathcal{D}} v-\varphi\right\|_{L^{\max (p, 2)}(\Omega)}+\left\|\nabla_{\mathcal{D}} v-\nabla \varphi\right\|_{L^{p}(\Omega)^{d}}\right)
$$

A sequence $\left(\mathcal{D}_{m}\right)_{m \in \mathbb{N}}$ of space-time gradient discretisations is said to be consistent if:

- for all $\varphi \in W_{0}^{1, p}(\Omega) \cap L^{2}(\Omega), \widehat{S}_{\mathcal{D}_{m}}(\varphi)$ tends to 0 as $m \rightarrow \infty$,

- $\delta t_{\mathcal{D}_{m}}$ tends to 0 as $m \rightarrow \infty$.

Theorem 4.7 (Convergence of the scheme) Under assumptions (3.4) and (4.1), let $\left(\mathcal{D}_{m}\right)_{m \in \mathbb{N}}$ be a sequence of space-time gradient discretisations in the sense of Definition 4.2, which is consistent (Definition 4.6) and such that the associated sequence of gradient discretisations is coercive (Definition 2.4), limit-conforming (Definition 2.7) and compact (Definition 2.8). Let $\alpha \in\left[\frac{1}{2}, 1\right]$ be given. For any $m \in \mathbb{N}$, let $u_{\mathcal{D}_{m}}$ be a solution to Scheme (4.3) with $u_{\mathcal{D}_{m}}^{(0)}$ chosen such that $\left\|u_{\mathrm{ini}}-\Pi_{\mathcal{D}_{m}} u_{\mathcal{D}_{m}}^{(0)}\right\|_{L^{2}(\Omega)} \rightarrow 0$ as $m \rightarrow \infty$. Then, up to a subsequence, $\Pi_{\mathcal{D}_{m}}^{(\alpha)} u_{\mathcal{D}_{m}}$ converges strongly in $L^{1}\left(0, T ; L^{p}(\Omega)\right)$ and in $L^{2}(\Omega \times(0, T))$ to a solution $\bar{u}$ of $(4.2), \Pi_{\mathcal{D}_{m}}^{(1)} u_{\mathcal{D}_{m}}$ converges strongly in $L^{2}(\Omega \times(0, T))$ to $\bar{u}$ and $\nabla_{\mathcal{D}_{m}} u_{\mathcal{D}_{m}}$ converges weakly in $L^{p}(\Omega \times(0, T))^{d}$ to $\nabla \bar{u}$ as $m \rightarrow \infty$.

Moreover, if we assume that the Leray-Lions operator $\boldsymbol{a}$ is strictly monotone in the sense of (3.7), then $\nabla_{\mathcal{D}_{m}} u_{\mathcal{D}_{m}}$ converges strongly in $L^{p}(\Omega \times(0, T))^{d}$ to $\nabla \bar{u}$ and $\Pi_{\mathcal{D}_{m}}^{(\alpha)} u_{\mathcal{D}_{m}}$ converges strongly in $L^{p}(\Omega \times(0, T))$ to $\bar{u}$ as $m \rightarrow \infty$.

In the case where the solution $\bar{u}$ of (3.6) is unique, then the whole sequence converges to $\bar{u}$ as $m \rightarrow \infty$ in the senses above.

Remark 4.8 As for the stationary problem (see Remark 3.7), the existence of a solution to (4.2) is a by-product of the proof of this theorem.

Proof We shall simply denote by $u_{m}$ instead of $u_{\mathcal{D}_{m}}$ a solution to Scheme (4.3) using the space-time gradient discretisation $\mathcal{D}_{m}$. In this proof, some indices $m$ are omitted in the expressions which are developed.

Step 1 Proof that hypotheses (h1)-(h2)-(h3)-(h4) of Theorem 5.13 hold with $\alpha_{m}^{(n)}=\alpha$ and $v_{m}^{(n)}=$ $\Pi_{\mathcal{D}_{m}} u_{m}^{(n)}$, and consequences.

In our setting, the space $B$ of Theorem 5.13 is $L^{p}(\Omega)$. We take $B_{m}=\Pi_{\mathcal{D}_{m}}\left(X_{\mathcal{D}_{m}, 0}\right)$. We define the norm $\|\cdot\|_{X_{m}}$ by

$$
\|v\|_{X_{m}}=\inf \left\{\|w\|_{\mathcal{D}_{m}}, w \in X_{\mathcal{D}, 0} \text { such that } \Pi_{\mathcal{D}_{m}} w=v\right\}
$$

(note that, for all $v \in B_{m}$, there exists one and only one $w \in X_{\mathcal{D}_{m}, 0}$ such that $\Pi_{\mathcal{D}_{m}} w=v$ and $\|w\|_{\mathcal{D}_{m}}=$ $\left.\|v\|_{X_{m}}\right)$ and the norm $\|\cdot\|_{Y_{m}}$ is defined from Definition 4.4 by

$$
\begin{aligned}
\|v\|_{Y_{m}} & =|w|_{\star, \mathcal{D}_{m}} \text { for any } w \in X_{\mathcal{D}_{m}, 0} \text { such that } \Pi_{\mathcal{D}_{m}} w=v \\
& =\sup \left\{\int_{\Omega} v(\boldsymbol{x}) \Pi_{\mathcal{D}_{m}} z(\boldsymbol{x}) \mathrm{d} \boldsymbol{x}, z \in X_{\mathcal{D}_{m}, 0},\|z\|_{\mathcal{D}_{m}}=1\right\} .
\end{aligned}
$$

We remark that $\|\cdot\|_{Y_{m}}$ is indeed a norm (if $v \neq 0$, then $v=\Pi_{\mathcal{D}_{m}} w$ with $w \neq 0$, and taking $z=w /\|w\|_{\mathcal{D}_{m}}$ shows that $\left.\|v\|_{Y_{m}}>0\right)$.

Let $\left(v_{m}\right)_{m \in \mathbb{N}}$ be a sequence of functions of $B_{m}$ such that $\left\|v_{m}\right\|_{X_{m}} \leq C$ for some $C \in \mathbb{R}_{+}$. Then, taking $w_{m} \in X_{\mathcal{D}_{m}, 0}$ such that $v_{m}=\Pi_{\mathcal{D}_{m}}\left(w_{m}\right)$ and $\left\|v_{m}\right\|_{X_{m}}=\left\|w_{m}\right\|_{\mathcal{D}_{m}}$, we get that the norm $\left\|w_{m}\right\|_{\mathcal{D}_{m}}$ remains bounded. Thanks to the coercivity and the compactness of the sequence of discretisations, a subsequence of $\left(\Pi_{\mathcal{D}_{m}} w_{m}\right)_{m \in \mathbb{N}}$ converges in $L^{p}(\Omega)$ to some $v \in L^{p}(\Omega)$. Thus, assumption (h1) of Theorem 5.13 is satisfied. 
Let us then show that assumption (h2) is also satisfied. Let $\left(v_{m}\right)_{m \in \mathbb{N}}$ be a sequence of functions of $L^{p}(\Omega)$ such that $v_{m} \in B_{m},\left\|v_{m}\right\|_{X_{m}} \leq C$ for some $C \in \mathbb{R}_{+}$, and such that there exists $v \in B$ with $v_{m} \rightarrow v$ in $B$ and $\left\|v_{m}\right\|_{Y_{m}} \rightarrow 0$ as $m \rightarrow \infty$. Taking $w_{m} \in X_{\mathcal{D}_{m}, 0}$ such that $v_{m}=\Pi_{\mathcal{D}_{m}}\left(w_{m}\right)$ and $\left\|v_{m}\right\|_{X_{m}}=\left\|w_{m}\right\|_{\mathcal{D}_{m}}$, we have

$$
\int_{\Omega}\left(v_{m}(\boldsymbol{x})\right)^{2} \mathrm{~d} \boldsymbol{x}=\int_{\Omega}\left(\Pi_{\mathcal{D}_{m}} w_{m}(\boldsymbol{x})\right)^{2} \mathrm{~d} \boldsymbol{x} \leq\left|w_{m}\right|_{\star, \mathcal{D}_{m}}\left\|w_{m}\right\|_{\mathcal{D}_{m}} \leq C\left\|v_{m}\right\|_{Y_{m}} \rightarrow 0 \text { as } m \rightarrow \infty
$$

which shows that $v=0$.

Estimates (4.4) and (4.6) show that hypotheses (h3) and (h4) of Theorem 5.13 are satisfied as well. Therefore, we deduce that there exists $\bar{u} \in L^{1}\left(0, T ; L^{p}(\Omega)\right)$ and a subsequence of $\left(\mathcal{D}_{m}\right)_{m \in \mathbb{N}}$, again denoted by $\left(\mathcal{D}_{m}\right)_{m \in \mathbb{N}}$, such that $\Pi_{\mathcal{D}_{m}}^{(\alpha)} u_{m}$ converges in $L^{1}\left(0, T ; L^{p}(\Omega)\right)$ to $\bar{u}$ as $m \rightarrow \infty$.

Step 2 Convergence of $\Pi_{\mathcal{D}_{m}}^{(1)} u_{m}$ and $\nabla_{\mathcal{D}_{m}} u_{m}$.

Thanks to Lemma 4.3 , the convergence of $\Pi_{\mathcal{D}_{m}}^{(\alpha)} u_{m}$ to $\bar{u}$ also holds in $L^{\infty}\left(0, T ; L^{2}(\Omega)\right)$ weak-*. The same lemma allows us to assume that $\Pi_{\mathcal{D}_{m}}^{(1)} u_{m}$ converges for the weak-* topology of $L^{\infty}\left(0, T ; L^{2}(\Omega)\right)$. Let us take $\varphi \in C_{c}^{\infty}(\Omega \times(0, T))$, define

$$
P_{\mathcal{D}} v=\underset{w \in X_{\mathcal{D}, 0}}{\operatorname{argmin}}\left(\left\|\Pi_{\mathcal{D}} w-v\right\|_{L^{\max (p, 2)}(\Omega)}+\left\|\nabla_{\mathcal{D}} w-\nabla v\right\|_{L^{p}(\Omega) d}\right), \forall v \in W_{0}^{1, p}(\Omega) \cap L^{2}(\Omega)
$$

and let $P_{\mathcal{D}} \varphi(t)=P_{\mathcal{D}}(\varphi(\cdot, t))$. Using the fact that $0 \in X_{\mathcal{D}_{m}, 0}$, we get that $\widehat{S}_{\mathcal{D}}(\varphi(t)) \leq\|\varphi(t)\|_{L^{\max (p, 2)}(\Omega)}+$ $\|\nabla \varphi(t)\|_{L^{p}(\Omega)^{d}}$, which leads, thanks to the triangular inequality, to

$$
\left\|\Pi_{\mathcal{D}_{m}} P_{\mathcal{D}_{m}} \varphi(t)\right\|_{L^{\max (p, 2)}(\Omega)}+\left\|P_{\mathcal{D}_{m}} \varphi(t)\right\|_{\mathcal{D}_{m}} \leq 2\left(\|\varphi(t)\|_{L^{\max (p, 2)}(\Omega)}+\|\nabla \varphi(t)\|_{L^{p}(\Omega)^{d}}\right) .
$$

We then write

$$
\begin{aligned}
& \left|\int_{0}^{T} \int_{\Omega}\left(\Pi_{\mathcal{D}_{m}}^{(1)} u_{m}(\boldsymbol{x}, t)-\Pi_{\mathcal{D}_{m}}^{(\alpha)} u_{m}(\boldsymbol{x}, t)\right) \Pi_{\mathcal{D}_{m}} P_{\mathcal{D}_{m}} \varphi(\boldsymbol{x}, t) \mathrm{d} \boldsymbol{x} \mathrm{d} t\right| \\
& \leq(1-\alpha) \delta t_{\mathcal{D}_{m}} \int_{0}^{T}\left|\delta_{\mathcal{D}_{m}} u(t)\right|_{\star, \mathcal{D}_{m}}\left\|P_{\mathcal{D}_{m}} \varphi(t)\right\|_{\mathcal{D}_{m}} \mathrm{~d} t
\end{aligned}
$$

and use Lemma 4.5 and Estimate (4.9) to see that the right-hand side of this inequality tends to 0 as $m \rightarrow \infty$. Thanks to the consistency of the sequence of approximations, for all $t \in \mathbb{R}$ we have $\Pi_{\mathcal{D}_{m}} P_{\mathcal{D}_{m}} \varphi(t) \rightarrow \varphi(t)$ in $L^{2}(\Omega)$ as $m \rightarrow \infty$. Hence, the dominated convergence theorem and (4.9) show that $\Pi_{\mathcal{D}_{m}} P_{\mathcal{D}_{m}} \varphi \rightarrow \varphi$ in $L^{2}\left(0, T ; L^{2}(\Omega)\right)$. Since $\Pi_{\mathcal{D}_{m}}^{(\alpha)} u_{m}$ and $\Pi_{\mathcal{D}_{m}}^{(1)} u_{m}$ are bounded in $L^{2}\left(0, T ; L^{2}(\Omega)\right)$, we deduce

$$
\begin{aligned}
& 0=\lim _{m \rightarrow \infty} \int_{0}^{T} \int_{\Omega}\left(\Pi_{\mathcal{D}_{m}}^{(1)} u_{m}(\boldsymbol{x}, t)-\Pi_{\mathcal{D}_{m}}^{(\alpha)} u_{m}(\boldsymbol{x}, t)\right) \Pi_{\mathcal{D}_{m}} P_{\mathcal{D}_{m}} \varphi(\boldsymbol{x}, t) \mathrm{d} \boldsymbol{x} \mathrm{d} t \\
& =\lim _{m \rightarrow \infty} \int_{0}^{T} \int_{\Omega}\left(\Pi_{\mathcal{D}_{m}}^{(1)} u_{m}(\boldsymbol{x}, t)-\Pi_{\mathcal{D}_{m}}^{(\alpha)} u_{m}(\boldsymbol{x}, t)\right) \varphi(\boldsymbol{x}, t) \mathrm{d} \boldsymbol{x} \mathrm{d} t .
\end{aligned}
$$

This proves that the weak-* limits of $\Pi_{\mathcal{D}_{m}}^{(1)} u_{m}$ and $\Pi_{\mathcal{D}_{m}}^{(\alpha)} u_{m}$ in $L^{\infty}\left(0, T ; L^{2}(\Omega)\right)$ are identical (equal to $\bar{u}$ ). By (4.4) and the coercivity of the sequence of gradient discretisations, we also have $\Pi_{\mathcal{D}_{m}}^{(\alpha)} u_{m} \rightarrow \bar{u}$ weakly in $L^{p}\left(0, T ; L^{p}(\Omega)\right)$ and we can assume that $\nabla_{\mathcal{D}_{m}} u_{m}$ converges weakly to some $\overline{\boldsymbol{G}}$ in $L^{p}\left(0, T ; L^{p}(\Omega)^{d}\right)$ as $m \rightarrow \infty$. Extending $\Pi_{\mathcal{D}_{m}}^{(\alpha)} u_{m}, \nabla_{\mathcal{D}_{m}} u_{m}, \bar{u}$ and $\overline{\boldsymbol{G}}$ by 0 outside $\Omega$, the consistency of the underlying gradient discretisation gives, as in the proof of Theorem 3.6, $\overline{\boldsymbol{G}}=\nabla \bar{u}$ and $\bar{u} \in L^{p}\left(0, T ; W_{0}^{1, p}(\Omega)\right)$. Finally, we notice that, by Assumption (3.4d), the functions $\mathcal{A}_{\mathcal{D}_{m}}(\boldsymbol{x}, t)=\boldsymbol{a}\left(\boldsymbol{x}, \Pi_{\mathcal{D}_{m}}^{(\alpha)} u_{m}(\cdot, t), \nabla_{\mathcal{D}_{m}} u_{m}(\boldsymbol{x}, t)\right)$ remain bounded in $L^{p^{\prime}}(\Omega \times(0, T))^{d}$ and converges therefore, up to a subsequence, to some $\boldsymbol{A}$ weakly in $L^{p^{\prime}}(\Omega \times(0, T))^{d}$ as $m \rightarrow \infty$.

Step 3 Proof that $\bar{u}$ is solution to (4.2). 
Let $\varphi \in C_{c}^{1}(-\infty, T)$ and $v \in W_{0}^{1, p}(\Omega) \cap L^{2}(\Omega)$. We introduce $\delta t^{\left(n+\frac{1}{2}\right)} \varphi\left(t^{(n)}\right) P_{\mathcal{D}} v$ as test function in (4.3). Summing on $n$ we get $T_{1}^{(m)}+T_{2}^{(m)}=T_{3}^{(m)}$, with

$$
\begin{gathered}
T_{1}^{(m)}=\sum_{n=0}^{N-1} \varphi\left(t^{(n)}\right) \delta t^{\left(n+\frac{1}{2}\right)} \int_{\Omega} \Pi_{\mathcal{D}} \delta_{\mathcal{D}}^{\left(n+\frac{1}{2}\right)} u(\boldsymbol{x}) \Pi_{\mathcal{D}} P_{\mathcal{D}} v(\boldsymbol{x}) \mathrm{d} \boldsymbol{x} \\
T_{2}^{(m)}=\sum_{n=0}^{N-1} \varphi\left(t^{(n)}\right) \delta t^{\left(n+\frac{1}{2}\right)} \int_{\Omega} \boldsymbol{a}\left(\boldsymbol{x}, \Pi_{\mathcal{D}} u^{(n+\alpha)}, \nabla_{\mathcal{D}} u^{(n+\alpha)}(\boldsymbol{x})\right) \cdot \nabla_{\mathcal{D}} P_{\mathcal{D}} v(\boldsymbol{x}) \mathrm{d} \boldsymbol{x} \\
T_{3}^{(m)}=\sum_{n=0}^{N-1} \varphi\left(t^{(n)}\right) \int_{t^{(n)}}^{t^{(n+1)}} \int_{\Omega} f(\boldsymbol{x}, t) \Pi_{\mathcal{D}} P_{\mathcal{D}} v(\boldsymbol{x}) \mathrm{d} \boldsymbol{x} \mathrm{d} t
\end{gathered}
$$

Using discrete integrate-by-parts formula to transform the terms $\varphi\left(t^{(n)}\right)\left(\Pi_{\mathcal{D}} u^{(n+1)}-\Pi_{\mathcal{D}} u^{(n)}\right)$ appearing in $T_{1}^{(m)}$ into $\left(\varphi\left(t^{(n)}\right)-\varphi\left(t^{(n+1)}\right)\right) \Pi_{\mathcal{D}} u^{(n+1)}$, we have

$$
T_{1}^{(m)}=-\int_{0}^{T} \varphi^{\prime}(t) \int_{\Omega} \Pi_{\mathcal{D}}^{(1)} u(\boldsymbol{x}, t) \Pi_{\mathcal{D}} P_{\mathcal{D}} v(\boldsymbol{x}) \mathrm{d} \boldsymbol{x} \mathrm{d} t-\varphi(0) \int_{\Omega} \Pi_{\mathcal{D}} u^{(0)}(\boldsymbol{x}) \Pi_{\mathcal{D}} P_{\mathcal{D}} v(\boldsymbol{x}) \mathrm{d} \boldsymbol{x} .
$$

Letting $\varphi_{\mathcal{D}}(t)=\varphi\left(t^{(n)}\right)$ for $t \in\left(t^{(n)}, t^{(n+1)}\right)$, we have

$$
T_{2}^{(m)}=\int_{0}^{T} \varphi_{\mathcal{D}}(t) \int_{\Omega} \mathcal{A}_{\mathcal{D}}(\boldsymbol{x}, t) \cdot \nabla_{\mathcal{D}} P_{\mathcal{D}} v(\boldsymbol{x}) \mathrm{d} \boldsymbol{x} \mathrm{d} t, \quad T_{3}^{(m)}=\int_{0}^{T} \varphi_{\mathcal{D}}(t) \int_{\Omega} f(\boldsymbol{x}, t) \Pi_{\mathcal{D}} P_{\mathcal{D}} v(\boldsymbol{x}) \mathrm{d} \boldsymbol{x} \mathrm{d} t .
$$

We may then let $m \rightarrow \infty$ and see that $\bar{u}$ satisfies

$$
\left\{\begin{array}{l}
\bar{u} \in L^{p}\left(0, T ; W_{0}^{1, p}(\Omega)\right) \cap L^{\infty}\left(0, T ; L^{2}(\Omega)\right), \\
-\int_{0}^{T} \varphi^{\prime}(t) \int_{\Omega} \bar{u}(\boldsymbol{x}, t) v(\boldsymbol{x}) \mathrm{d} \boldsymbol{x} \mathrm{d} t-\varphi(0) \int_{\Omega} u_{\mathrm{ini}}(\boldsymbol{x}) v(\boldsymbol{x}) \mathrm{d} \boldsymbol{x} \\
+\int_{0}^{T} \varphi(t) \int_{\Omega} \boldsymbol{A}(\boldsymbol{x}, t) \cdot \nabla v(\boldsymbol{x}) \mathrm{d} \boldsymbol{x} \mathrm{d} t=\int_{0}^{T} \varphi(t) \int_{\Omega} f(\boldsymbol{x}, t) v(\boldsymbol{x}) \mathrm{d} \boldsymbol{x} \mathrm{d} t \\
\forall v \in W_{0}^{1, p}(\Omega) \cap L^{2}(\Omega), \forall \varphi \in C_{c}^{\infty}(-\infty, T) .
\end{array}\right.
$$

Linear combinations of this relation show that it also holds with $\varphi(t) v(\boldsymbol{x})$ replaced by a tensorial functions in $C_{c}^{\infty}(\Omega \times(0, T))$ and, by Remark 4.1, allows to prove that $\partial_{t} \bar{u} \in L^{p^{\prime}}\left(0, T ; W^{-1, p^{\prime}}(\Omega)\right)$. Standard arguments then show that $\bar{u}$ can be identified with an element of $C^{0}\left([0, T] ; L^{2}(\Omega)\right)$, with the property $\bar{u}(\cdot, 0)=u_{\mathrm{ini}}$. Using the density of tensorial functions in $L^{p}\left(0, T ; W_{0}^{1, p}(\Omega)\right)$, we then see that $\bar{u}$ satisfies

$$
\left\{\begin{array}{l}
\int_{0}^{T}\left\langle\partial_{t} \bar{u}(\cdot, t), \bar{v}(\cdot, t)\right\rangle_{W^{-1, p^{\prime}}(\Omega), W_{0}^{1, p}(\Omega)} \mathrm{d} t \\
+\int_{0}^{T} \int_{\Omega} \boldsymbol{A}(\boldsymbol{x}, t) \cdot \nabla \bar{v}(\boldsymbol{x}, t) \mathrm{d} \boldsymbol{x} \mathrm{d} t=\int_{0}^{T} \int_{\Omega} f(\boldsymbol{x}, t) \bar{v}(\boldsymbol{x}, t) \mathrm{d} \boldsymbol{x} \mathrm{d} t, \quad \forall \bar{v} \in L^{p}\left(0, T ; W_{0}^{1, p}(\Omega)\right) .
\end{array}\right.
$$

It remains to prove that

$$
\boldsymbol{A}(\boldsymbol{x}, t)=\boldsymbol{a}(\boldsymbol{x}, \bar{u}(\cdot, t), \nabla \bar{u}(\boldsymbol{x}, t)), \text { for a.e. }(\boldsymbol{x}, t) \in \Omega \times(0, T) .
$$

We start by writing (4.12) with $\bar{v}=\varphi(t) \bar{u}$, for a given $\varphi \in C_{c}^{\infty}(-\infty, T)$ such that $\varphi^{\prime}(t) \leq 0$ for all $t \geq 0$ (which implies $\varphi(t) \geq 0$ for all $t \geq 0$ ). Using

$$
\int_{0}^{T}\left\langle\partial_{t} \bar{u}(\cdot, t), \varphi(t) \bar{u}(\cdot, t)\right\rangle_{W^{-1, p^{\prime}}(\Omega), W_{0}^{1, p}(\Omega)} \mathrm{d} t=-\frac{1}{2} \int_{0}^{T} \varphi^{\prime}(t) \int_{\Omega} \bar{u}(\boldsymbol{x}, t)^{2} \mathrm{~d} \boldsymbol{x} \mathrm{d} t-\frac{1}{2} \varphi(0) \int_{\Omega} \bar{u}(\boldsymbol{x}, 0)^{2} \mathrm{~d} \boldsymbol{x},
$$


we get

$$
\left\{\begin{array}{l}
-\frac{1}{2} \int_{0}^{T} \varphi^{\prime}(t) \int_{\Omega} \bar{u}(\boldsymbol{x}, t)^{2} \mathrm{~d} \boldsymbol{x} \mathrm{d} t-\frac{1}{2} \varphi(0) \int_{\Omega} u_{\text {ini }}(\boldsymbol{x})^{2} \mathrm{~d} \boldsymbol{x} \\
+\int_{0}^{T^{T}} \varphi(t) \int_{\Omega} \boldsymbol{A}(\boldsymbol{x}, t) \cdot \nabla \bar{u}(\boldsymbol{x}, t) \mathrm{d} \boldsymbol{x} \mathrm{d} t=\int_{0}^{T} \varphi(t) \int_{\Omega} f(\boldsymbol{x}, t) \bar{u}(\boldsymbol{x}, t) \mathrm{d} \boldsymbol{x} \mathrm{d} t .
\end{array}\right.
$$

We then introduce the test function $v=\varphi\left(t^{(n)}\right) u^{(n+\alpha)}$ in (4.3), and we sum on $n=0, \ldots, N-1$. Since we have

$$
\begin{aligned}
& \sum_{n=0}^{N-1} \Pi_{\mathcal{D}}\left(u^{(n+1)}-u^{(n)}\right) \varphi\left(t^{(n)}\right) \Pi_{\mathcal{D}}\left(\alpha u^{(n+1)}+(1-\alpha) u^{(n)}\right)=-\frac{1}{2} \sum_{n=0}^{N-1}\left(\Pi_{\mathcal{D}} u^{(n+1)}\right)^{2}\left(\varphi\left(t^{(n+1)}\right)-\varphi\left(t^{(n)}\right)\right) \\
& -\frac{1}{2}\left(\Pi_{\mathcal{D}} u^{(0)}\right)^{2} \varphi(0)+\left(\alpha-\frac{1}{2}\right) \sum_{n=0}^{N-1}\left(\Pi_{\mathcal{D}} u^{(n+1)}-\Pi_{\mathcal{D}} u^{(n)}\right)^{2} \varphi\left(t^{(n)}\right)
\end{aligned}
$$

we may write, setting $\varphi_{\mathcal{D}}(t)=\varphi\left(t^{(n)}\right)$ for $t \in\left(t^{(n)}, t^{(n+1)}\right)$,

$$
\begin{aligned}
& -\frac{1}{2} \int_{0}^{T} \varphi^{\prime}(t) \int_{\Omega}\left(\Pi_{\mathcal{D}}^{(1)} u(\boldsymbol{x}, t)\right)^{2} \mathrm{~d} \boldsymbol{x} \mathrm{d} t-\frac{1}{2} \varphi(0) \int_{\Omega}\left(\Pi_{\mathcal{D}} u^{(0)}(\boldsymbol{x})\right)^{2} \mathrm{~d} \boldsymbol{x} \\
& +\int_{0}^{T} \varphi_{\mathcal{D}}(t) \int_{\Omega} \boldsymbol{a}\left(\boldsymbol{x}, \Pi_{\mathcal{D}}^{(\alpha)} u(\cdot, t), \nabla_{\mathcal{D}} u(\boldsymbol{x}, t)\right) \cdot \nabla_{\mathcal{D}} u(\boldsymbol{x}, t) \mathrm{d} \boldsymbol{x} \mathrm{d} t \\
& \leq \int_{0}^{T} \varphi_{\mathcal{D}}(t) \int_{\Omega} f(\boldsymbol{x}, t) \Pi_{\mathcal{D}}^{(\alpha)} u(\boldsymbol{x}, t) \mathrm{d} \boldsymbol{x} \mathrm{d} t .
\end{aligned}
$$

Since $\Pi_{\mathcal{D}_{m}}^{(1)} u_{m}$ converges to $\bar{u}$ weakly in $L^{2}(\Omega \times(0, T))$ and recalling that $\varphi^{\prime}(t) \leq 0$, we have

$$
\liminf _{m \rightarrow \infty}-\frac{1}{2} \int_{0}^{T} \varphi^{\prime}(t) \int_{\Omega}\left(\Pi_{\mathcal{D}_{m}}^{(1)} u_{m}(\boldsymbol{x}, t)\right)^{2} \mathrm{~d} \boldsymbol{x} \mathrm{d} t \geq-\frac{1}{2} \int_{0}^{T} \varphi^{\prime}(t) \int_{\Omega}(\bar{u}(\boldsymbol{x}, t))^{2} \mathrm{~d} \boldsymbol{x} \mathrm{d} t .
$$

This shows, thanks to (4.14), that

$$
\begin{aligned}
& \limsup _{m \rightarrow \infty} \int_{0}^{T} \varphi_{\mathcal{D}_{m}}(t) \int_{\Omega} \boldsymbol{a}\left(\boldsymbol{x}, \Pi_{\mathcal{D}_{m}}^{(\alpha)} u_{m}(\cdot, t), \nabla_{\mathcal{D}_{m}} u_{m}(\boldsymbol{x}, t)\right) \cdot \nabla_{\mathcal{D}_{m}} u_{m}(\boldsymbol{x}, t) \mathrm{d} \boldsymbol{x} \mathrm{d} t \\
& \quad \leq \int_{0}^{T} \varphi(t) \int_{\Omega} \boldsymbol{A}(\boldsymbol{x}, t) \cdot \nabla \bar{u}(\boldsymbol{x}, t) .
\end{aligned}
$$

It is now possible to apply Minty's trick. Considering, for $\boldsymbol{G} \in L^{p}\left(0, T ; L^{p}(\Omega)\right)^{d}$,

$$
\int_{0}^{T} \varphi_{\mathcal{D}}(t) \int_{\Omega}\left(\boldsymbol{a}\left(\boldsymbol{x}, \Pi_{\mathcal{D}}^{(\alpha)} u(\cdot, t), \nabla_{\mathcal{D}} u(\boldsymbol{x}, t)\right)-\boldsymbol{a}\left(\boldsymbol{x}, \Pi_{\mathcal{D}}^{(\alpha)} u(\cdot, t), \boldsymbol{G}(\boldsymbol{x}, t)\right)\right) \cdot\left(\nabla_{\mathcal{D}} u(\boldsymbol{x}, t)-\boldsymbol{G}(\boldsymbol{x}, t)\right) \mathrm{d} \boldsymbol{x} \mathrm{d} t \geq 0 .
$$

Developing this inequality and using (4.16) for the only "weak-weak" term, we may take the lim sup as $m \rightarrow \infty$ to find

$$
\int_{0}^{T} \varphi(t) \int_{\Omega}(\boldsymbol{A}(\boldsymbol{x}, t)-\boldsymbol{a}(\boldsymbol{x}, \bar{u}(\cdot, t), \boldsymbol{G}(\boldsymbol{x}, t))) \cdot(\nabla \bar{u}(\boldsymbol{x}, t)-\boldsymbol{G}(\boldsymbol{x}, t)) \mathrm{d} \boldsymbol{x} \geq 0, \forall \boldsymbol{G} \in L^{p}\left(0, T ; L^{p}(\Omega)^{d}\right) .
$$

By taking $\varphi$ which approximates the characteristic function of $(-\infty, T)$, we can remove it from this equation. Application of Minty's method then shows that (4.13) holds and concludes the proof that $\bar{u}$ is a weak solution to (4.2).

Step 4 Strong $L^{2}(\Omega \times(0, T))$-convergence of the approximate solution.

In view of $(3.4 \mathrm{c})$ and (4.16) we have

$$
\begin{aligned}
& \lim _{m \rightarrow \infty} \int_{0}^{T} \varphi_{\mathcal{D}}(t) \int_{\Omega}\left(\boldsymbol{a}\left(\boldsymbol{x}, \Pi_{\mathcal{D}}^{(\alpha)} u(\cdot, t), \nabla_{\mathcal{D}} u_{\mathcal{D}}(\boldsymbol{x}, t)\right)-\boldsymbol{a}\left(\boldsymbol{x}, \Pi_{\mathcal{D}}^{(\alpha)} u(\cdot, t), \nabla \bar{u}(\boldsymbol{x}, t)\right)\right) \\
& \cdot\left(\nabla_{\mathcal{D}} u_{\mathcal{D}}(\boldsymbol{x}, t)-\nabla \bar{u}(\boldsymbol{x}, t)\right) \mathrm{d} \boldsymbol{x} \mathrm{d} t=0,
\end{aligned}
$$


which proves, using (4.13), that

$$
\begin{aligned}
& \lim _{m \rightarrow \infty} \int_{0}^{T} \varphi_{\mathcal{D}_{m}}(t) \int_{\Omega} \boldsymbol{a}\left(\boldsymbol{x}, \Pi_{\mathcal{D}_{m}}^{(\alpha)} u(\cdot, t), \nabla_{\mathcal{D}_{m}} u_{m}(\boldsymbol{x}, t)\right) \cdot \nabla_{\mathcal{D}_{m}} u_{m}(\boldsymbol{x}, t) \mathrm{d} \boldsymbol{x} \mathrm{d} t \\
& =\int_{0}^{T} \varphi(t) \int_{\Omega} \boldsymbol{a}(\boldsymbol{x}, \bar{u}(\cdot, t), \nabla \bar{u}(\boldsymbol{x}, t)) \cdot \nabla \bar{u}(\boldsymbol{x}, t) \mathrm{d} \boldsymbol{x} \mathrm{d} t .
\end{aligned}
$$

As a result, from (4.14), (4.15) and (4.18) and letting $\varphi(t) \rightarrow T-t$, we obtain

$$
\limsup _{m \rightarrow \infty} \frac{1}{2} \int_{0}^{T} \int_{\Omega}\left(\Pi_{\mathcal{D}_{m}}^{(1)} u_{m}(\boldsymbol{x}, t)\right)^{2} \mathrm{~d} \boldsymbol{x} \mathrm{d} t \leq \frac{1}{2} \int_{0}^{T} \int_{\Omega}(\bar{u}(\boldsymbol{x}, t))^{2} \mathrm{~d} \boldsymbol{x} \mathrm{d} t
$$

which shows that the weak convergence of $\Pi_{\mathcal{D}_{m}}^{(1)} u_{m}$ to $\bar{u}$ in $L^{2}(\Omega \times(0, T))$ is in fact strong. We then remark that

$$
\begin{aligned}
& \left|\int_{0}^{T} \int_{\Omega}\left(\Pi_{\mathcal{D}_{m}}^{(\alpha)} u_{m}(\boldsymbol{x}, t)\right)^{2} \mathrm{~d} \boldsymbol{x} \mathrm{d} t-\int_{0}^{T} \int_{\Omega} \Pi_{\mathcal{D}_{m}}^{(1)} u_{m}(\boldsymbol{x}, t) \Pi_{\mathcal{D}_{m}}^{(\alpha)} u_{m}(\boldsymbol{x}, t) \mathrm{d} \boldsymbol{x} \mathrm{d} t\right| \\
& \leq(1-\alpha) \delta t_{\mathcal{D}_{m}} \int_{0}^{T}\left|\delta_{\mathcal{D}_{m}} u_{m}(t)\right|_{\star, \mathcal{D}_{m}}\left\|u_{m}(\cdot, t)\right\|_{\mathcal{D}_{m}} \mathrm{~d} t \\
& \leq(1-\alpha) \delta t_{\mathcal{D}_{m}}\left(\int_{0}^{T}\left|\delta_{\mathcal{D}_{m}} u_{m}(t)\right|_{\star, \mathcal{D}_{m}}^{p^{\prime}}\right)^{1 / p^{\prime}}\left\|\nabla_{\mathcal{D}_{m}} u_{m}\right\|_{L^{p}(\Omega \times(0, T))^{d}} .
\end{aligned}
$$

By Lemmas 4.3 and 4.5, the right-hand side tends to 0 as $m \rightarrow \infty$. Moreover, by strong/weak convergence we have

$$
\lim _{m \rightarrow \infty} \int_{0}^{T} \int_{\Omega} \Pi_{\mathcal{D}_{m}}^{(1)} u_{m}(\boldsymbol{x}, t) \Pi_{\mathcal{D}_{m}}^{(\alpha)} u_{m}(\boldsymbol{x}, t) \mathrm{d} \boldsymbol{x} \mathrm{d} t=\int_{0}^{T} \int_{\Omega}(\bar{u}(\boldsymbol{x}, t))^{2} \mathrm{~d} \boldsymbol{x} \mathrm{d} t .
$$

Therefore,

$$
\lim _{m \rightarrow \infty} \int_{0}^{T} \int_{\Omega}\left(\Pi_{\mathcal{D}_{m}}^{(\alpha)} u_{m}(\boldsymbol{x}, t)\right)^{2} \mathrm{~d} \boldsymbol{x} \mathrm{d} t=\int_{0}^{T} \int_{\Omega}(\bar{u}(\boldsymbol{x}, t))^{2} \mathrm{~d} \boldsymbol{x} \mathrm{d} t
$$

and the weak convergence of $\Pi_{\mathcal{D}_{m}}^{(\alpha)} u_{m}$ to $\bar{u}$ in $L^{2}(\Omega \times(0, T))$ is in fact also strong.

Step 5 Assuming now hypothesis (3.7), strong convergence of the approximate gradient.

Using (4.17), the proof is similar to that of Theorem 3.6.

Remark 4.9 Due to the generic form of $\boldsymbol{a}$ and the very limited number of discrete properties we require on gradient schemes to prove their convergence, we cannot provide an error estimate similar to the one in Lemma 3.1. One would expect such an error estimate to be expressed in terms of the $L^{\infty}\left(0, T ; L^{2}(\Omega)\right)$ norm and to give, in particular, convergence in this space.

The following can however be noticed. In the continuous case, the $L^{\infty}\left(0, T ; L^{2}(\Omega)\right)$ norm of a function can be estimated using its $L^{2}(\Omega)$ norm at $t=0$, its $L^{p}\left(0, T ; W_{0}^{1, p}(\Omega)\right)$ norm and the $L^{p^{\prime}}\left(0, T ; W^{-1, p^{\prime}}(\Omega)\right)$ norm of its time derivative. A similar estimate can be written, using the dual semi-norm from Definition 4.4, in the setting of gradient schemes: for all $v:[0, T] \rightarrow X_{\mathcal{D}, 0}$ piecewise constant equal to $v^{(n)}$ on $\left(t^{(n)}, t^{(n+1)}\right)$, we have

$$
\forall t \in(0, T):\left\|\Pi_{\mathcal{D}} v(t)\right\|_{L^{2}(\Omega)}^{2} \leq\left\|\Pi_{\mathcal{D}} v(0)\right\|_{L^{2}(\Omega)}^{2}+2\left(\int_{0}^{T}\left|\delta_{\mathcal{D}} v(t)\right|_{\star, \mathcal{D}}^{p^{\prime}} d t\right)^{1 / p^{\prime}}\left\|\nabla_{\mathcal{D}} v\right\|_{L^{p}(\Omega \times(0, T))^{d}}
$$

(this is proved by writing $\left(\Pi_{\mathcal{D}} v^{(n+1)}\right)^{2}-\left(\Pi_{\mathcal{D}} v^{(n)}\right)^{2}=\left(\Pi_{\mathcal{D}} v^{(n+1)}+\Pi_{\mathcal{D}} v^{(n)}\right) \delta t^{\left(n+\frac{1}{2}\right)} \Pi_{\mathcal{D}} \delta_{\mathcal{D}}^{\left(n+\frac{1}{2}\right)} v$ and summing on $n)$. Hence, if we assume the existence of an interpolant $Q_{\mathcal{D}_{m}} \bar{u}:[0, T] \rightarrow X_{\mathcal{D}_{m}, 0}$ of the exact solution, 
which is constant on each $\left(t^{(n)}, t^{(n+1)}\right)$ and such that, as $m \rightarrow \infty$,

$$
\begin{aligned}
& Q_{\mathcal{D}_{m}} \bar{u} \rightarrow \bar{u} \text { in } L^{\infty}\left(0, T ; L^{2}(\Omega)\right), \nabla_{\mathcal{D}_{m}} Q_{\mathcal{D}_{m}} \bar{u} \rightarrow \nabla u \text { in } L^{p}(\Omega \times(0, T))^{d} \text { and } \\
& \left(\int_{0}^{T}\left|\delta_{\mathcal{D}_{m}} Q_{\mathcal{D}_{m}} \bar{u}(t)\right|_{\star, \mathcal{D}}^{p^{\prime}} d t\right)_{m \in \mathbb{N}} \text { remains bounded, }
\end{aligned}
$$

applying (4.19) to $v=u_{\mathcal{D}_{m}}-Q_{\mathcal{D}_{m}} \bar{u}$ proves that if $\boldsymbol{a}$ is strictly monotone (and therefore $\nabla_{\mathcal{D}_{m}} u_{\mathcal{D}_{m}} \rightarrow \nabla \bar{u}$ strongly) then the convergence of $\Pi_{\mathcal{D}_{m}} u_{\mathcal{D}_{m}}$ to $\bar{u}$ also holds in $L^{\infty}\left(0, T ; L^{2}(\Omega)\right)$.

Under some regularity assumptions on $\bar{u}$, the existence of $Q_{\mathcal{D}_{m}} \bar{u}$ is clearly true for all gradient schemes considered in the introduction.

\section{The Hybrid Mimetic Mixed family of schemes}

We proved in [18] that three families of numerical methods independently developed for the linear problem (3.1), namely the SUSHI scheme [20], the Mimetic Finite Difference methods [7, 8] and the Mixed Finite Volume methods [16, 17], can all be gathered under a same generalising framework, the Hybrid Mimetic Mixed (HMM) methods. Some adaptations of these methods to nonlinear equations and systems have been proposed in $[15,11,19]$, but they involve $a d$-hoc (and arguably cumbersome) stabilisations and do not fully take advantage, as in Section 3 and 4 above, of coercive gradients coming from gradient schemes. It was however proved in [21] that one of the HMM methods, the SUSHI scheme, can be written as a gradient scheme for linear equations.

We show in this section that, in fact, any scheme of the HMM family can be viewed as a gradient scheme for the linear problem (3.1), for gradient discretisations which are coercive, consistent, limitconforming and compact. The work of Sections 3 and 4 therefore allows for a very natural generalisation of HMM methods to the nonlocal nonlinear elliptic and parabolic problems (1.1) and (1.2), using in the formulations (2.1) and (4.3) the gradient discretisations coming from the HMM methods for linear problems. This is probably the most natural and efficient adaptation of HMM methods to nonlinear problems, since it involves a natural coercive gradient and does not require the introduction of artificial stabilisation terms as in previous works.

In this section, we assume $\Omega$ to be an open polygonal bounded and connected subset of $\mathbb{R}^{d}$ with Lipschitzcontinuous boundary $\partial \Omega$.

\subsection{Polygonal meshes}

Let us first give the definition of the meshes which are used for the HMM family, see Figure 1 for some notations.

Definition 5.1 (Pointed strictly star-shaped polygonal mesh) A pointed strictly star-shaped polygonal mesh of $\Omega$ is given by the triplet $(\mathcal{M}, \mathcal{E}, \mathcal{P})$, where:

1. $\mathcal{M}$ is a finite family of non empty connected open disjoint subsets of $\Omega$ (the "control volumes" or "cells") such that $\bar{\Omega}=\cup_{K \in \mathcal{M}} \bar{K}$. For any $K \in \mathcal{M}$, let $\partial K=\bar{K} \backslash K$ be the boundary of $K,|K|>0$ be the measure of $K$ and $h_{K}$ be the diameter of $K$.

2. $\mathcal{E}=\mathcal{E}_{\text {int }} \cup \mathcal{E}_{\text {ext }}$ is a finite family of disjoint subsets of $\bar{\Omega}$ (the "edges" of the mesh) such that any $\sigma \in \mathcal{E}$ is a non-empty open subset of an hyperplane of $\mathbb{R}^{d}$. We denote by $\mathcal{E}_{\text {int }}$ the set of edges included in $\Omega$ and by $\mathcal{E}_{\mathrm{ext}}$ the set of edges included in $\partial \Omega$. The $(d-1)$-dimensional measure and the center of gravity of $\sigma \in \mathcal{E}$ are respectively denoted by $|\sigma|$ and $\overline{\boldsymbol{x}}_{\sigma}$.

We assume that, for all $K \in \mathcal{M}$, there exists a subset $\mathcal{E}_{K}$ of $\mathcal{E}$ such that $\partial K=\cup_{\sigma \in \mathcal{E}_{K}} \bar{\sigma}$. We then set $\mathcal{M}_{\sigma}=\left\{K \in \mathcal{M}, \sigma \in \mathcal{E}_{K}\right\}$ and we assume that, for all $\sigma \in \mathcal{E}$, either $\mathcal{M}_{\sigma}$ has exactly one element and then $\sigma \in \mathcal{E}_{\text {ext }}$ or $\mathcal{M}_{\sigma}$ has exactly two elements and then $\sigma \in \mathcal{E}_{\text {int }}$. For all $K \in \mathcal{M}$ and any $\sigma \in \mathcal{E}_{K}$, we denote by $\boldsymbol{n}_{K, \sigma}$ the unit vector normal to $\sigma$ outward to $K$. 


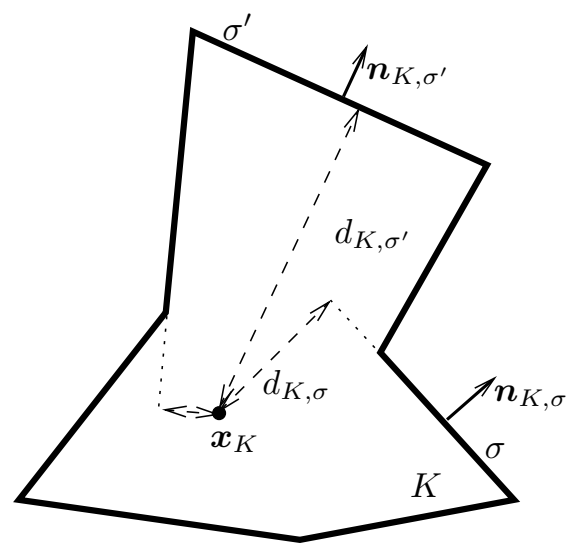

Figure 1: A control volume $K$ of a pointed strictly star-shaped polygonal mesh

3. $\mathcal{P}=\left(\boldsymbol{x}_{K}\right)_{K \in \mathcal{M}}$ is a family of points of $\Omega$ indexed by $\mathcal{M}$ such that, for all $K \in \mathcal{M}, \boldsymbol{x}_{K} \in K$. We then denote by $d_{K, \sigma}$ the signed distance between $\boldsymbol{x}_{K}$ and $\sigma$ (see Figure 1), that is:

$$
d_{K, \sigma}=\left(\boldsymbol{x}-\boldsymbol{x}_{K}\right) \cdot \boldsymbol{n}_{K, \sigma}, \quad \boldsymbol{x} \in \sigma .
$$

(Note that $\left(\boldsymbol{x}-\boldsymbol{x}_{K}\right) \cdot \boldsymbol{n}_{K, \sigma}$ is constant for $\boldsymbol{x} \in \sigma$.)

We further assume that all cells $K \in \mathcal{M}$ are strictly $\boldsymbol{x}_{K}$-star-shaped, which means that, for all $\boldsymbol{x} \in K$, the line segment $\left[\boldsymbol{x}_{K}, \boldsymbol{x}\right]$ is included in $K$ or, equivalently, that $d_{K, \sigma}>0$ for all $\sigma \in \mathcal{E}_{K}$.

The size of the discretisation is defined by $h_{\mathcal{D}}=\max \left\{h_{K}, K \in \mathcal{M}\right\}$. For all $K \in \mathcal{M}$ and $\sigma \in \mathcal{E}_{K}$, we denote by $D_{K, \sigma}$ the cone with vertex $\boldsymbol{x}_{K}$ and basis $\sigma: D_{K, \sigma}=\left\{t \boldsymbol{x}_{K}+(1-t) \boldsymbol{y}, t \in(0,1), \boldsymbol{y} \in \sigma\right\}$. We have

$$
\left|D_{K, \sigma}\right|=\frac{1}{d}|\sigma| d_{K, \sigma} \quad \text { and } \quad \sum_{\sigma \in \mathcal{E}_{K}}|\sigma| d_{K, \sigma}=d|K| .
$$

The following lemma, which directly results from the Stokes formula, is classically used in the construction of consistent approximate gradients.

Lemma 5.2 Let $K$ be a nonempty polyhedral subset of $\mathbb{R}^{d}$. For $\sigma \in \mathcal{E}_{K}$, we denote by $\overline{\boldsymbol{x}}_{\sigma}$ the barycentre of $\sigma$ and by $\boldsymbol{n}_{K, \sigma}$ the unit vector normal to $\sigma$ outward to $K$. Let $\boldsymbol{x}_{K}$ be any point of $\mathbb{R}^{d}$. Then:

$$
\sum_{\sigma \in \mathcal{E}_{K}}|\sigma| \boldsymbol{n}_{K, \sigma}\left(\overline{\boldsymbol{x}}_{\sigma}-\boldsymbol{x}_{K}\right)^{t}=|K| \mathrm{Id},
$$

where $\left(\overline{\boldsymbol{x}}_{\sigma}-\boldsymbol{x}_{K}\right)^{t}$ is the transpose of $\overline{\boldsymbol{x}}_{\sigma}-\boldsymbol{x}_{K} \in \mathbb{R}^{d}$ and Id is the $d \times d$ identity matrix.

\subsection{Hybrid Mimetic Mixed methods}

There are several equivalent implementations of HMM methods for linear problems. The one presented here, which allows to interpret all HMM methods as gradient schemes, uses "pressure" unknowns on the faces of the mesh (other implementations use flux unknowns on the faces).

Let $(\mathcal{M}, \mathcal{E}, \mathcal{P})$ be a strictly star-shaped pointed polygonal mesh of $\Omega$, in the sense of Definition 5.1. Let

$$
X_{\mathcal{D}, 0}=\left\{v=\left(\left(v_{K}\right)_{K \in \mathcal{M}},\left(v_{\sigma}\right)_{\sigma \in \mathcal{E}}\right): v_{K} \in \mathbb{R}, v_{\sigma} \in \mathbb{R}, v_{\sigma}=0 \text { for all } \sigma \in \mathcal{E}_{\text {ext }}\right\},
$$

and define, for $v \in X_{\mathcal{D}, 0}, K \in \mathcal{M}$ and $\sigma \in \mathcal{E}_{K}$,

$$
\nabla_{K} v=\frac{1}{|K|} \sum_{\sigma \in \mathcal{E}_{K}}|\sigma|\left(v_{\sigma}-v_{K}\right) \boldsymbol{n}_{K, \sigma} \quad \text { and } \quad R_{K, \sigma}(v)=v_{\sigma}-v_{K}-\nabla_{K} v \cdot\left(\overline{\boldsymbol{x}}_{\sigma}-\boldsymbol{x}_{K}\right) .
$$


We also define $R_{K}(v)=\left(R_{K, \sigma}(v)\right)_{\sigma \in \mathcal{E}_{K}}$ and $\Lambda_{K}=$ value of $\Lambda$ on $K(\Lambda$ is assumed to be constant in each cell). With these notations, any Hybrid Mimetic Mixed method for Problem (3.1) can be written

$$
\begin{aligned}
& \text { Find } u \in X_{\mathcal{D}, 0} \text { such that, for all } v \in X_{\mathcal{D}, 0}, \\
& \sum_{K \in \mathcal{M}}|K| \Lambda_{K} \nabla_{K} u \cdot \nabla_{K} v+\sum_{K \in \mathcal{M}} R_{K}(v)^{T} \mathbb{B}_{K} R_{K}(u)=\sum_{K \in \mathcal{M}} v_{K} \int_{K} f(\boldsymbol{x}) \mathrm{d} \boldsymbol{x}
\end{aligned}
$$

with $\mathbb{B}_{K} \in \mathbb{R}^{\mathcal{E}_{K} \times \mathcal{E}_{K}}$ symmetric positive definite matrices with suitable properties (see Remark 5.7).

Remark 5.3 (A generalisation with barycentric edge unknowns) We could, as in [20], express some values $v_{\sigma}$ as barycentric combinations of the values $v_{K}$, thus obtaining a cell-centred scheme. All the properties given here can be extended to this case.

\subsection{All HMM methods are gradient schemes}

\subsubsection{Definition of the gradient discretisation}

Let $\Pi_{\mathcal{D}}: X_{\mathcal{D}, 0} \rightarrow L^{2}(\Omega)$ be defined by

$$
\forall v \in X_{\mathcal{D}, 0}, \forall K \in \mathcal{M}, \Pi_{\mathcal{D}}(v)=v_{K} \text { on } K .
$$

The right-hand side of (5.5) is therefore equal to $\int_{\Omega} f \Pi_{\mathcal{D}} v$. In order to prove that any HMM method is a gradient scheme we now have to find, for any choice of $\left(\mathbb{B}_{K}\right)_{K \in \mathcal{M}}$, a discrete gradient $\nabla_{\mathcal{D}}$ such that, for all $(u, v) \in X_{\mathcal{D}, 0}^{2}$,

$$
\sum_{K \in \mathcal{M}}|K| \Lambda_{K} \nabla_{K} u \cdot \nabla_{K} v+\sum_{K \in \mathcal{M}} R_{K}(v)^{T} \mathbb{B}_{K} R_{K}(u)=\int_{\Omega} \Lambda(\boldsymbol{x}) \nabla_{\mathcal{D}} u(\boldsymbol{x}) \cdot \nabla_{\mathcal{D}} v(\boldsymbol{x}) \mathrm{d} \boldsymbol{x} .
$$

For any $v \in X_{\mathcal{D}, 0}$, we look for a piecewise constant gradient $\nabla_{\mathcal{D}} v$ defined by:

$$
\forall K \in \mathcal{M}, \forall \sigma \in \mathcal{E}_{K}, \forall \boldsymbol{x} \in D_{K, \sigma}: \nabla_{\mathcal{D}} v(\boldsymbol{x})=\nabla_{K, \sigma} v:=\nabla_{K} v+\frac{\sqrt{d}}{d_{K, \sigma}}\left(A_{K} \widetilde{R}_{K}\left(V_{K}\right)\right)_{\sigma} \boldsymbol{n}_{K, \sigma},
$$

where

- $V_{K}=\left(v_{\sigma}-v_{K}\right)_{\sigma \in \mathcal{E}_{K}}$,

- $\widetilde{R}_{K}: \mathbb{R}^{\mathcal{E}_{K}} \rightarrow \mathbb{R}^{\mathcal{E}_{K}}$ is the linear mapping defined by $\widetilde{R}_{K}(\xi)=\left(\widetilde{R}_{K, \sigma}(\xi)\right)_{\sigma \in \mathcal{E}_{K}}$ with

$$
\widetilde{R}_{K, \sigma}(\xi)=\xi_{\sigma}-\left(\frac{1}{|K|} \sum_{\sigma^{\prime} \in \mathcal{E}_{K}}\left|\sigma^{\prime}\right| \xi_{\sigma^{\prime}} \boldsymbol{n}_{K, \sigma^{\prime}}\right) \cdot\left(\overline{\boldsymbol{x}}_{\sigma}-\boldsymbol{x}_{K}\right),
$$

- $A_{K}$ is an isomorphism, to be defined, of the vector space $\operatorname{Im}\left(\widetilde{R}_{K}\right) \subset \mathbb{R}^{\mathcal{E}_{K}}$.

We prove below that, for any symmetric positive definite matrix $\mathbb{B}_{K} \in \mathbb{R}^{\mathcal{E}_{K} \times \mathcal{E}_{K}}$, we can find $A_{K}$ such that for all $(u, v) \in X_{\mathcal{D}, 0}^{2}$,

$$
\sum_{\sigma \in \mathcal{E}_{K}}\left|D_{K, \sigma}\right| \Lambda_{K} \nabla_{K, \sigma} u \cdot \nabla_{K, \sigma} v=|K| \Lambda_{K} \nabla_{K} u \cdot \nabla_{K} v+R_{K}(v)^{T} \mathbb{B}_{K} R_{K}(u) .
$$

With such $A_{K}$ 's, the gradient $\nabla_{\mathcal{D}}$ defined by (5.8) satisfies (5.7) and the HMM method (5.5) is exactly the gradient scheme given by the gradient discretisation $\mathcal{D}=\left(X_{\mathcal{D}, 0}, \Pi_{\mathcal{D}}, \nabla_{\mathcal{D}}\right)$.

To find $A_{K}: \operatorname{Im}\left(\widetilde{R}_{K}\right) \rightarrow \operatorname{Im}\left(\widetilde{R}_{K}\right)$ we first notice that, by Lemma 5.2 , the linear mapping

$$
G_{K}: \eta \in \mathbb{R}^{\mathcal{E}_{K}} \rightarrow \sum_{\sigma \in \mathcal{E}_{K}}|\sigma| \eta_{\sigma} \boldsymbol{n}_{K, \sigma} \in \mathbb{R}^{d}
$$


vanishes on $\operatorname{Im}\left(\widetilde{R}_{K}\right)$. Since $A_{K}$ takes its values in this space, using (5.2) we deduce

$$
\forall \xi \in \mathbb{R}^{\mathcal{E}_{K}}, \sum_{\sigma \in \mathcal{E}_{K}}\left|D_{K, \sigma}\right| \frac{\sqrt{d}}{d_{K, \sigma}}\left(A_{K} \widetilde{R}_{K}(\xi)\right)_{\sigma} \boldsymbol{n}_{K, \sigma}=\frac{1}{\sqrt{d}} \sum_{\sigma \in \mathcal{E}_{K}}|\sigma|\left(A_{K} \widetilde{R}_{K}(\xi)\right)_{\sigma} \boldsymbol{n}_{K, \sigma}=0 .
$$

Hence, from the definition (5.8) and since $\sum_{\sigma \in \mathcal{E}_{K}}\left|D_{K, \sigma}\right|=|K|$, we infer

$$
\begin{aligned}
& \sum_{\sigma \in \mathcal{E}_{K}} \mid D_{K, \sigma} \mid \Lambda_{K} \nabla_{K, \sigma} u \cdot \nabla_{K, \sigma} v \\
&=|K| \Lambda_{K} \nabla_{K} u \cdot \nabla_{K} v+\sum_{\sigma \in \mathcal{E}_{K}}\left|D_{K, \sigma}\right| \frac{d}{d_{K, \sigma}^{2}} \Lambda_{K} \boldsymbol{n}_{K, \sigma} \cdot \boldsymbol{n}_{K, \sigma}\left(A_{K} \widetilde{R}_{K}\left(U_{K}\right)\right)_{\sigma}\left(A_{K} \widetilde{R}_{K}\left(V_{K}\right)\right)_{\sigma} \\
& \quad=|K| \Lambda_{K} \nabla_{K} u \cdot \nabla_{K} v+\left(A_{K} \widetilde{R}_{K}\left(V_{K}\right)\right)^{T} \mathbb{D}_{K}\left(A_{K} \widetilde{R}_{K}\left(U_{K}\right)\right)
\end{aligned}
$$

where $U_{K}=\left(u_{\sigma}-u_{K}\right)_{\sigma \in \mathcal{E}_{K}}$ and $\mathbb{D}_{K}=\operatorname{diag}\left(\frac{|\sigma|}{d_{K, \sigma}} \Lambda_{K} \boldsymbol{n}_{K, \sigma} \cdot \boldsymbol{n}_{K, \sigma}\right)$ is diagonal definite positive. Since $R_{K}(u)=\widetilde{R}_{K}\left(U_{K}\right)$ and $R_{K}(v)=\widetilde{R}_{K}\left(V_{K}\right),(5.9)$ is satisfied provided that, for all $(\xi, \eta) \in\left(\mathbb{R}^{\mathcal{E}_{K}}\right)^{2}$,

$$
\left(A_{K} \widetilde{R}_{K}(\xi)\right)^{T} \mathbb{D}_{K}\left(A_{K} \widetilde{R}_{K}(\eta)\right)=\widetilde{R}_{K}(\xi)^{T} \mathbb{B}_{K} \widetilde{R}_{K}(\eta) .
$$

We now apply Lemma 5.4 below with $E=\operatorname{Im}\left(\widetilde{R}_{K}\right) \subset \mathbb{R}^{\mathcal{E}_{K}}$ and the inner products $\langle x, y\rangle_{1}=y^{T} \mathbb{B}_{K} x$ and $\langle x, y\rangle_{2}=y^{T} \mathbb{D}_{K} x$. The isomorphism $A_{K}: \operatorname{Im}\left(\widetilde{R}_{K}\right) \rightarrow \operatorname{Im}\left(\widetilde{R}_{K}\right)$ given by Lemma 5.4 satisfies (5.13) which, applied with $x=\widetilde{R}_{K}(\eta)$ and $y=\widetilde{R}_{K}(\xi)$, is precisely $(5.12)$.

Lemma 5.4 Let $E$ be a finite-dimensional vector space endowed with two inner products $\langle,\rangle_{1}$ and $\langle,\rangle_{2}$. Then there exists an isomorphism $A: E \rightarrow E$ such that

$$
\text { for all }(x, y) \in E^{2},\langle x, y\rangle_{1}=\langle A x, A y\rangle_{2} \text {. }
$$

Proof Let $e$ be an orthonormal basis for $\langle,\rangle_{2}$ and $M_{e}$ be the (symmetric definite positive) matrix of $\langle,\rangle_{1}$ in this basis. If $X_{e}$ and $Y_{e}$ are the coordinates of $x$ and $y$ in $e$ then $\langle x, y\rangle_{1}=Y_{e}^{T} M_{e} X_{e}$. Let then $A_{e}=\sqrt{M_{e}}$ and define $A$ as the isomorphism whose matrix relative to the basis $e$ is $A_{e}$. Since $e$ is orthonormal for $\langle,\rangle_{2}$, the relation $Y_{e}^{T} M_{e} X_{e}=\left(A_{e} Y_{e}\right)^{T}\left(A_{e} X_{e}\right)$ translates into $\langle x, y\rangle_{1}=\langle A x, A y\rangle_{2}$.

Remark 5.5 Since $\left(\boldsymbol{n}_{K, \sigma}\right)_{\sigma \in \mathcal{E}_{K}}$ spans $\mathbb{R}^{d}$, the mapping $G_{K}$ defined by (5.10) has rank d, which implies $\operatorname{dim}\left(\operatorname{ker} G_{K}\right)=\operatorname{Card}\left(\mathcal{E}_{K}\right)-d$. It is easy to see that $\operatorname{ker}\left(\widetilde{R}_{K}\right)=\left\{\xi \in \mathbb{R}^{\mathcal{E}_{K}} ; \exists Z_{\xi} \in \mathbb{R}^{d}\right.$ such that $\xi_{\sigma}=$ $\left.Z_{\xi} \cdot\left(\overline{\boldsymbol{x}}_{\sigma}-\boldsymbol{x}_{K}\right)\right\}$ and thus that $Z \in \mathbb{R}^{d} \rightarrow\left(Z \cdot\left(\overline{\boldsymbol{x}}_{\sigma}-\boldsymbol{x}_{K}\right)\right)_{\sigma \in \mathcal{E}_{K}} \in \operatorname{ker}\left(\widetilde{R}_{K}\right)$ is an isomorphism (the one to one property comes from the fact that $\left(\overline{\boldsymbol{x}}_{\sigma}-\boldsymbol{x}_{K}\right)_{\sigma \in \mathcal{E}_{K}}$ spans $\left.\mathbb{R}^{d}\right)$. Hence, $\operatorname{dim}\left(\operatorname{Im}\left(\widetilde{R}_{K}\right)\right)=\operatorname{Card}\left(\mathcal{E}_{K}\right)-d=$ $\left.\operatorname{dim}\left(\operatorname{ker}\left(G_{K}\right)\right)\right)$. Since $\operatorname{Im}\left(\widetilde{R}_{K}\right) \subset \operatorname{ker}\left(G_{K}\right)$, we infer that $\operatorname{Im}\left(\widetilde{R}_{K}\right)=\operatorname{ker}\left(G_{K}\right)$.

Thus, $A_{K}$ can be indifferently searched as an isomorphism of $\operatorname{Im}\left(\widetilde{R}_{K}\right)$ or as an isomorphism of $\operatorname{ker}\left(G_{K}\right)$.

\subsubsection{Coercivity, consistency, limit-conformity and compactness}

We prove here that a gradient discretisation corresponding to an HMM method is, under the usual assumptions on HMM methods, coercive, consistent, limit-conforming and compact. The proof is based on the characterisation of these properties given in Lemma 2.9.

Lemma 5.6 (Coercivity) Let $p \in[1,+\infty),(\mathcal{M}, \mathcal{E}, \mathcal{P})$ be a strictly star-shaped pointed polygonal mesh of $\Omega$ in the sense of Definition 5.1 and let $\left(X_{\mathcal{D}, 0}, \Pi_{\mathcal{D}}, \nabla_{\mathcal{D}}\right)$ be given by (5.4), (5.6) and (5.8), for some $\left(A_{K}\right)_{K \in \mathcal{M}}$. We take $\theta>0$ such that

$$
\max \left(\max _{\sigma \in \mathcal{E}_{\text {int }}, K, L \in \mathcal{M}_{\sigma}} \frac{d_{K, \sigma}}{d_{L, \sigma}}, \max _{K \in \mathcal{M}, \sigma \in \mathcal{E}_{K}} \frac{h_{K}}{d_{K, \sigma}}\right) \leq \theta
$$


and

$$
\begin{aligned}
& \forall K \in \mathcal{M}, \forall \xi \in \mathbb{R}^{\mathcal{E}_{K}}, \\
& \frac{1}{\theta} \sum_{\sigma \in \mathcal{E}_{K}}\left|D_{K, \sigma}\right|\left|\frac{\widetilde{R}_{K, \sigma}(\xi)}{d_{K, \sigma}}\right|^{p} \leq \sum_{\sigma \in \mathcal{E}_{K}}\left|D_{K, \sigma}\right|\left|\frac{\left(A_{K} \widetilde{R}_{K}(\xi)\right)_{\sigma}}{d_{K, \sigma}}\right|^{p} \leq \theta \sum_{\sigma \in \mathcal{E}_{K}}\left|D_{K, \sigma}\right|\left|\frac{\widetilde{R}_{K, \sigma}(\xi)}{d_{K, \sigma}}\right|^{p} .
\end{aligned}
$$

Then there exists $C_{5}>1$ only depending on $\Omega, p$ and $\theta$ such that:

$$
\frac{1}{C_{5}}\|u\|_{1, p} \leq\left\|\nabla_{\mathcal{D}} u\right\|_{L^{p}(\Omega)} \leq C_{5}\|u\|_{1, p} \quad \forall u \in X_{\mathcal{D}, 0},
$$

where we have taken

$$
\|u\|_{1, p}^{p}=\sum_{K \in \mathcal{M}} \sum_{\sigma \in \mathcal{E}_{K}}|\sigma| d_{K, \sigma}\left|\frac{u_{\sigma}-u_{K}}{d_{K, \sigma}}\right|^{p} .
$$

Consequently, there exists $C_{6}>0$ only depending on $\Omega, p$ and $\theta$ such that:

$$
C_{\mathcal{D}} \leq C_{6},
$$

where $C_{\mathcal{D}}$ is defined by $(2.2)$.

Remark 5.7 To ensure the convergence of the HMM method, matrices $\mathbb{B}_{K}$ are assumed to satisfy the following (see [18]): there exist $s_{*}>0$ and $S_{*}>0$ independent of the mesh such that, for all $K \in \mathcal{M}$ and all $\xi \in \mathbb{R}^{\mathcal{E}_{K}}$,

$$
s_{*} \sum_{\sigma \in \mathcal{E}_{K}} \frac{|\sigma|}{d_{K, \sigma}}\left(\widetilde{R}_{K, \sigma}(\xi)\right)^{2} \leq \widetilde{R}_{K}(\xi)^{T} \mathbb{B}_{K} \widetilde{R}_{K}(\xi) \leq S_{*} \sum_{\sigma \in \mathcal{E}_{K}} \frac{|\sigma|}{d_{K, \sigma}}\left(\widetilde{R}_{K, \sigma}(\xi)\right)^{2} .
$$

If $A_{K}$ is chosen so that (5.12) holds then, since $\mathbb{D}_{K}=\operatorname{diag}\left(\frac{|\sigma|}{d_{K, \sigma}} \Lambda_{K} \boldsymbol{n}_{K, \sigma} \cdot \boldsymbol{n}_{K, \sigma}\right)$, there exists $C_{7}$ only depending on $d, s_{*}, S_{*}, \underline{\lambda}$ and $\bar{\lambda}$ (see (3.2)) such that

$$
\frac{1}{C_{7}} \sum_{\sigma \in \mathcal{E}_{K}} \frac{|\sigma|}{d_{K, \sigma}}\left(\widetilde{R}_{K, \sigma}(\xi)\right)^{2} \leq \sum_{\sigma \in \mathcal{E}_{K}} \frac{|\sigma|}{d_{K, \sigma}}\left(A_{K} \widetilde{R}_{K}(\xi)\right)_{\sigma}^{2} \leq C_{7} \sum_{\sigma \in \mathcal{E}_{K}} \frac{|\sigma|}{d_{K, \sigma}}\left(\widetilde{R}_{K, \sigma}(\xi)\right)^{2}
$$

which is precisely (5.15) for $p=2$. Hence, in the linear framework (the only one in which generic HMM methods have been defined up to now), the gradient scheme corresponding to an HMM method satisfies the assumptions in Lemma 5.6 with $\theta$ not depending on the mesh.

Remark 5.8 An easy way to choose $A_{K}$ such that (5.15) holds is to take $A_{K}=\beta_{K} \operatorname{Id}_{\operatorname{Im}\left(\widetilde{R}_{K}\right)}$ for some $\beta_{K} \in\left[\frac{1}{\theta}, \theta\right]$. The corresponding HMM method is then the SUSHI scheme of [20].

\section{Proof}

In this proof, the notation $\mathcal{A}(u) \sim \mathcal{B}(u)$ means that there exists $C$ only depending on $\Omega, p$ and $\theta$ such that, for all $u \in X_{\mathcal{D}, 0}, C^{-1} \mathcal{A}(u) \leq \mathcal{B}(u) \leq C \mathcal{A}(u)$.

The key ingredient in the proof is to notice that $\nabla_{K} u$ is a convex combination of $\left(\nabla_{K, \sigma} u\right)_{\sigma \in \mathcal{E}_{K}}$. Precisely, (5.11) shows that $\nabla_{K} u=\sum_{\sigma \in \mathcal{E}_{K}} \frac{\left|D_{K, \sigma}\right|}{|K|} \nabla_{K, \sigma} u$. By convexity of $|\cdot|^{p}$ on $\mathbb{R}^{d}$, we infer

$$
\left|\nabla_{K} u\right|^{p} \leq \sum_{\sigma \in \mathcal{E}_{K}} \frac{\left|D_{K, \sigma}\right|}{|K|}\left|\nabla_{K, \sigma} u\right|^{p} .
$$

From $\sqrt{d} \frac{\left(A_{K} \widetilde{R}_{K}\left(U_{K}\right)\right)_{\sigma}}{d_{K, \sigma}} \boldsymbol{n}_{K, \sigma}=\nabla_{K, \sigma} u-\nabla_{K} u$, we also have

$$
d^{p / 2} \sum_{\sigma \in \mathcal{E}_{K}}\left|D_{K, \sigma}\right|\left|\frac{\left(A_{K} \widetilde{R}_{K}\left(U_{K}\right)\right)_{\sigma}}{d_{K, \sigma}}\right|^{p} \leq 2^{p-1}|K|\left|\nabla_{K} u\right|^{p}+2^{p-1} \sum_{\sigma \in \mathcal{E}_{K}}\left|D_{K, \sigma}\right|\left|\nabla_{K, \sigma} u\right|^{p} .
$$


Together with (5.15), Estimates (5.18) and (5.19) show that

$$
|K|\left|\nabla_{K} u\right|^{p}+\sum_{\sigma \in \mathcal{E}_{K}}\left|D_{K, \sigma}\right|\left|\frac{\widetilde{R}_{K, \sigma}\left(U_{K}\right)}{d_{K, \sigma}}\right|^{p} \sim \sum_{\sigma \in \mathcal{E}_{K}}\left|D_{K, \sigma}\right|\left|\nabla_{K, \sigma} u\right|^{p} .
$$

Since $\frac{\widetilde{R}_{K, \sigma}\left(U_{K}\right)}{d_{K, \sigma}}=\frac{u_{\sigma}-u_{K}}{d_{K, \sigma}}-\nabla_{K} u \cdot \frac{\overline{\boldsymbol{x}}_{\sigma}-\boldsymbol{x}_{K}}{d_{K, \sigma}}$ and $\frac{\left|\overline{\boldsymbol{x}}_{\sigma}-\boldsymbol{x}_{K}\right|}{d_{K, \sigma}} \leq \theta$ thanks to (5.14), we see that

$$
|K|\left|\nabla_{K} u\right|^{p}+\sum_{\sigma \in \mathcal{E}_{K}}\left|D_{K, \sigma}\right|\left|\frac{\widetilde{R}_{K, \sigma}\left(U_{K}\right)}{d_{K, \sigma}}\right|^{p} \sim|K|\left|\nabla_{K} u\right|^{p}+\sum_{\sigma \in \mathcal{E}_{K}}\left|D_{K, \sigma}\right|\left|\frac{u_{\sigma}-u_{K}}{d_{K, \sigma}}\right|^{p}
$$

Finally, the definition of $\nabla_{K} u$ and (5.2) show that $\nabla_{K} u=d \sum_{\sigma \in \mathcal{E}_{K}} \frac{\left|D_{K, \sigma}\right|}{|K|} \frac{u_{\sigma}-u_{K}}{d_{K, \sigma}} \boldsymbol{n}_{K, \sigma}$ and thus that

$$
\left|\nabla_{K} u\right|^{p} \leq d^{p} \sum_{\sigma \in \mathcal{E}_{K}} \frac{\left|D_{K, \sigma}\right|}{|K|}\left|\frac{u_{\sigma}-u_{K}}{d_{K, \sigma}}\right|^{p}
$$

Since $\left\|\nabla_{\mathcal{D}} u\right\|_{L^{p}(\Omega)^{d}}^{p}=\sum_{K \in \mathcal{M}} \sum_{\sigma \in \mathcal{E}_{K}}\left|D_{K, \sigma}\right|\left|\nabla_{K, \sigma} u\right|^{p}$, Estimate (5.16) follows from (5.20), (5.21) and (5.22). To deduce (5.17) from (5.16), we notice that, whenever $\sigma$ is a common edge between $K$ and $L$,

$$
\frac{\left|u_{L}-u_{K}\right|}{d_{K, \sigma}+d_{L, \sigma}} \leq \frac{\left|u_{L}-u_{\sigma}\right|}{d_{L, \sigma}}+\frac{\left|u_{\sigma}-u_{K}\right|}{d_{K, \sigma}}
$$

and we conclude by [20, Lemma 5.4].

Lemma 5.9 (Limit-conformity) Let $p \in[1,+\infty),(\mathcal{M}, \mathcal{E}, \mathcal{P})$ be a strictly star-shaped pointed polygonal mesh of $\Omega$ in the sense of Definition 5.1 and let $\left(X_{\mathcal{D}, 0}, \Pi_{\mathcal{D}}, \nabla_{\mathcal{D}}\right)$ be given by (5.4), (5.6) and (5.8), for some $\left(A_{K}\right)_{K \in \mathcal{M}}$. We take $\theta>0$ such that (5.14) and (5.15) hold.

Then there exists $C_{8}$ only depending on $\Omega, p$ and $\theta$ such that

$$
W_{\mathcal{D}}(\boldsymbol{\varphi}) \leq C_{8} h_{\mathcal{D}}\|\varphi\|_{\left(W^{1, \infty}\left(\mathbb{R}^{d}\right)\right)^{d}}, \forall \boldsymbol{\varphi} \in\left(C_{c}^{\infty}\left(\mathbb{R}^{d}\right)\right)^{d} .
$$

Proof Let $\varphi \in C_{c}^{\infty}\left(\mathbb{R}^{d}\right)^{d}$ and $u \in X_{\mathcal{D}, 0}$. Let us define the terms $T_{1}^{\mathcal{D}}$ and $T_{2}^{\mathcal{D}}$ by

$$
T_{1}^{\mathcal{D}}=\int_{\mathbb{R}^{d}} \nabla_{\mathcal{D}} u_{\mathcal{D}}(\boldsymbol{x}) \cdot \boldsymbol{\varphi}(\boldsymbol{x}) \mathrm{d} \boldsymbol{x} \quad \text { and } \quad T_{2}^{\mathcal{D}}=-\int_{\mathbb{R}^{d}} \Pi_{\mathcal{D}} u_{\mathcal{D}}(\boldsymbol{x}) \operatorname{div} \boldsymbol{\varphi}(\boldsymbol{x}) \mathrm{d} \boldsymbol{x} .
$$

Since $\boldsymbol{n}_{K, \sigma}=-\boldsymbol{n}_{L, \sigma}$ whenever $\sigma$ is an edge between $K$ and $L$, and since $u_{\sigma}=0$ if $\sigma \in \mathcal{E}_{\text {ext }}$, letting $\boldsymbol{\varphi}_{\sigma}=\frac{1}{|\sigma|} \int_{\sigma} \boldsymbol{\varphi}(\boldsymbol{x}) \mathrm{d} \gamma(\boldsymbol{x})$ we have

$$
T_{2}^{\mathcal{D}}=\sum_{K \in \mathcal{M}} \sum_{\sigma \in \mathcal{E}_{K}}|\sigma|\left(u_{\sigma}-u_{K}\right) \boldsymbol{n}_{K, \sigma} \cdot \boldsymbol{\varphi}_{\sigma}
$$

By (5.8), we have $T_{1}^{\mathcal{D}}=T_{3}^{\mathcal{D}}+T_{4}^{\mathcal{D}}$ with

$T_{3}^{\mathcal{D}}=\sum_{K \in \mathcal{M}} \sum_{\sigma \in \mathcal{E}_{K}}|\sigma|\left(u_{\sigma}-u_{K}\right) \boldsymbol{n}_{K, \sigma} \cdot \boldsymbol{\varphi}_{K} \quad$ and $\quad T_{4}^{\mathcal{D}}=\sum_{K \in \mathcal{M}} \sum_{\sigma \in \mathcal{E}_{K}} \frac{\sqrt{d}}{d_{K, \sigma}}\left(A_{K} \widetilde{R}_{K}\left(U_{K}\right)\right)_{\sigma} \boldsymbol{n}_{K, \sigma} \cdot \int_{D_{K, \sigma}} \boldsymbol{\varphi}(\boldsymbol{x}) \mathrm{d} \boldsymbol{x}$ where $\varphi_{K}=\frac{1}{|K|} \int_{K} \boldsymbol{\varphi}(\boldsymbol{x}) \mathrm{d} \boldsymbol{x}$. Since $\left|\boldsymbol{\varphi}_{K}-\boldsymbol{\varphi}_{\sigma}\right| \leq h_{\mathcal{D}}\|\boldsymbol{\varphi}\|_{\left(W^{1, \infty}\left(\mathbb{R}^{d}\right)\right)^{d}}$ whenever $\sigma \in \mathcal{E}_{K}$, using Hölder's inequality we get $\left|T_{3}^{\mathcal{D}}-T_{2}^{\mathcal{D}}\right| \leq h_{\mathcal{D}}\|\varphi\|_{\left(W^{1, \infty}\left(\mathbb{R}^{d}\right)\right)^{d}}\|u\|_{1, p}(d|\Omega|)^{(p-1) / p}$. By (5.16), we therefore find $C_{9}$ only depending on $\Omega, p$ and $\theta$ such that

$$
\left|T_{3}^{\mathcal{D}}-T_{2}^{\mathcal{D}}\right| \leq C_{9} h_{\mathcal{D}}\|\varphi\|_{\left(W^{1, \infty}\left(\mathbb{R}^{d}\right)\right)^{d}}\left\|\nabla_{\mathcal{D}} u\right\|_{L^{p}(\Omega)^{d}}
$$


Invoking (5.11), we see that

$$
T_{4}^{\mathcal{D}}=\sum_{K \in \mathcal{M}} \sum_{\sigma \in \mathcal{E}_{K}} \frac{\sqrt{d}}{d_{K, \sigma}}\left(A_{K} \widetilde{R}_{K}\left(U_{K}\right)\right)_{\sigma} \boldsymbol{n}_{K, \sigma} \cdot \int_{D_{K, \sigma}}\left(\boldsymbol{\varphi}(\boldsymbol{x})-\boldsymbol{\varphi}_{K}\right) \mathrm{d} \boldsymbol{x} .
$$

Since $\left|\int_{D_{K, \sigma}}\left(\boldsymbol{\varphi}(\boldsymbol{x})-\boldsymbol{\varphi}_{K}\right) \mathrm{d} \boldsymbol{x}\right| \leq h_{\mathcal{D}}\|\boldsymbol{\varphi}\|_{\left(W^{1, \infty}\left(\mathbb{R}^{d}\right)\right)^{d}}\left|D_{K, \sigma}\right|$, this leads to

$$
\begin{aligned}
\left|T_{4}^{\mathcal{D}}\right| & \leq \sqrt{d} h_{\mathcal{D}}\|\varphi\|_{\left(W^{1, \infty}\left(\mathbb{R}^{d}\right)\right)^{d}} \sum_{K \in \mathcal{M}} \sum_{\sigma \in \mathcal{E}_{K}}\left|D_{K, \sigma}\right|\left|\frac{\left(A_{K} \widetilde{R}_{K}\left(U_{K}\right)\right)_{\sigma}}{d_{K, \sigma}}\right| \\
& \leq \sqrt{d}|\Omega|^{(p-1) / p} h_{\mathcal{D}}\|\boldsymbol{\varphi}\|_{\left(W^{1, \infty}\left(\mathbb{R}^{d}\right)\right)^{d}}\left(\sum_{K \in \mathcal{M}} \sum_{\sigma \in \mathcal{E}_{K}}\left|D_{K, \sigma}\right|\left|\frac{\left(A_{K} \widetilde{R}_{K}\left(U_{K}\right)\right)_{\sigma}}{d_{K, \sigma}}\right|^{p}\right)^{1 / p} .
\end{aligned}
$$

Recalling (5.15) and (5.20) in the proof of Lemma 5.6, we deduce the existence of $C_{10}$ only depending on $\Omega, p$ and $\theta$ such that

$$
\left|T_{4}^{\mathcal{D}}\right| \leq C_{10} h_{\mathcal{D}}\|\varphi\|_{\left(W^{1, \infty}\left(\mathbb{R}^{d}\right)\right)^{d}}\left\|\nabla_{\mathcal{D}} u\right\|_{L^{p}(\Omega)^{d}} .
$$

Equations (5.24) and (5.25) prove that there exists $C_{11}$ only depending on $\Omega, p$ and $\theta$ such that $\left|T_{1}^{\mathcal{D}}-T_{2}^{\mathcal{D}}\right|=$ $\left|T_{3}^{\mathcal{D}}-T_{2}^{\mathcal{D}}+T_{4}^{\mathcal{D}}\right| \leq C_{11} h_{\mathcal{D}}\|\boldsymbol{\varphi}\|_{\left(W^{1, \infty}\left(\mathbb{R}^{d}\right)\right)^{d}}\left\|\nabla_{\mathcal{D}} u\right\|_{L^{p}(\Omega)^{d}}$ and the proof is complete.

Lemma 5.10 (Consistency) Let $p \in[1,+\infty),(\mathcal{M}, \mathcal{E}, \mathcal{P})$ be a strictly star-shaped pointed polygonal mesh of $\Omega$ in the sense of Definition 5.1 and let $\left(X_{\mathcal{D}, 0}, \Pi_{\mathcal{D}}, \nabla_{\mathcal{D}}\right)$ be given by (5.4), (5.6) and (5.8), for some $\left(A_{K}\right)_{K \in \mathcal{M}}$. We take $\theta>0$ such that (5.14) and (5.15) hold.

Then there exists $C_{12}$ only depending on $\Omega, p$ and $\theta$ such that:

$$
S_{\mathcal{D}}(\varphi) \leq C h_{\mathcal{D}}\|\varphi\|_{W^{2, \infty}(\Omega)}, \forall \varphi \in C_{c}^{\infty}(\Omega) .
$$

Proof For all $\varphi \in C_{c}^{\infty}(\Omega)$, let $v \in X_{\mathcal{D}, 0}$ such that $v_{K}=\varphi\left(\boldsymbol{x}_{K}\right)$ for all $K \in \mathcal{M}$ and $v_{\sigma}=\varphi\left(\overline{\boldsymbol{x}}_{\sigma}\right)$ for all $\sigma \in \mathcal{E}$. We clearly have $\left\|\Pi_{\mathcal{D}} v-\varphi\right\|_{L^{\infty}(\Omega)} \leq h_{\mathcal{D}}\|\varphi\|_{W^{1, \infty}(\Omega)}$. Regarding the gradients, we first write

$$
\left|\nabla_{K, \sigma} v-\nabla \varphi\left(\boldsymbol{x}_{K}\right)\right| \leq\left|\nabla_{K} v-\nabla \varphi\left(\boldsymbol{x}_{K}\right)\right|+\left|\frac{\sqrt{d}}{d_{K, \sigma}}\left(A_{K} \widetilde{R}_{K}\left(V_{K}\right)\right)_{\sigma}\right| .
$$

Let $Z_{K, \sigma}=\varphi\left(\overline{\boldsymbol{x}}_{\sigma}\right)-\varphi\left(\boldsymbol{x}_{K}\right)-\nabla \varphi\left(\boldsymbol{x}_{K}\right) \cdot\left(\overline{\boldsymbol{x}}_{\sigma}-\boldsymbol{x}_{K}\right)$. We have, by Lemma 5.2,

$$
\begin{aligned}
\nabla \varphi\left(\boldsymbol{x}_{K}\right) & =\frac{1}{|K|} \sum_{\sigma \in \mathcal{E}_{K}}|\sigma|\left[\nabla \varphi\left(\boldsymbol{x}_{K}\right) \cdot\left(\overline{\boldsymbol{x}}_{\sigma}-\boldsymbol{x}_{K}\right)\right] \boldsymbol{n}_{K, \sigma} \\
& =\frac{1}{|K|} \sum_{\sigma \in \mathcal{E}_{K}}|\sigma|\left(\varphi\left(\overline{\boldsymbol{x}}_{\sigma}\right)-\varphi\left(\boldsymbol{x}_{K}\right)\right) \boldsymbol{n}_{K, \sigma}-\frac{1}{|K|} \sum_{\sigma \in \mathcal{E}_{K}}|\sigma| Z_{K, \sigma} \boldsymbol{n}_{K, \sigma} \\
& =\nabla_{K} v-\frac{1}{|K|} \sum_{\sigma \in \mathcal{E}_{K}}|\sigma| Z_{K, \sigma} \boldsymbol{n}_{K, \sigma} .
\end{aligned}
$$

Since $h_{K} \leq \theta d_{K, \sigma}$ and $\left|Z_{K, \sigma}\right| \leq h_{K}^{2}\|\varphi\|_{W^{2, \infty}(\Omega)}$, we deduce

$$
\left|\nabla \varphi\left(\boldsymbol{x}_{K}\right)-\nabla_{K} v\right| \leq d \theta h_{K}\|\varphi\|_{W^{2, \infty}(\Omega)} .
$$

We have $\widetilde{R}_{K, \sigma}\left(V_{K}\right)=v_{\sigma}-v_{K}-\nabla_{K} v \cdot\left(\overline{\boldsymbol{x}}_{\sigma}-\boldsymbol{x}_{K}\right)=Z_{K, \sigma}-\left(\nabla_{K} v-\nabla \varphi\left(\boldsymbol{x}_{K}\right)\right) \cdot\left(\overline{\boldsymbol{x}}_{\sigma}-\boldsymbol{x}_{K}\right)$ and thus $\left|\frac{\widetilde{R}_{K, \sigma}\left(V_{K}\right)}{d_{K, \sigma}}\right| \leq C_{13} h_{K}|| \varphi \|_{W^{2, \infty}(\Omega)}$ with $C_{13}$ only depending on $d, p$ and $\theta$. Using (5.15), we infer

$$
\sum_{\sigma \in \mathcal{E}_{K}}\left|D_{K, \sigma}\right|\left|\frac{\left(A_{K} \widetilde{R}_{K}\left(V_{K}\right)\right)_{\sigma}}{d_{K, \sigma}}\right|^{p} \leq \theta C_{13}^{p} h_{K}^{p}\|\varphi\|_{W^{2, \infty}(\Omega)}^{p}|K| .
$$

Using this estimate and (5.28) in (5.27) we obtain $\left\|\nabla_{\mathcal{D}} v-\nabla \varphi\right\|_{L^{p}(\Omega)} \leq C_{14} h_{\mathcal{D}}\|\varphi\|_{W^{2, \infty}(\Omega)}$ with $C_{14}$ only depending on $\Omega, p$ and $\theta$. The proof is complete. 
Remark 5.11 Since we estimated $\left\|\Pi_{\mathcal{D}} v-\varphi\right\|_{L^{\infty}(\Omega)}$ (and not only the $L^{p}$ norm), the same proof shows that the space-time consistency (Definition 4.6) also holds.

Lemma 5.12 (Compactness) Let $p \in[1,+\infty),(\mathcal{M}, \mathcal{E}, \mathcal{P})$ be a strictly star-shaped pointed polygonal mesh of $\Omega$ in the sense of Definition 5.1 and let $\left(X_{\mathcal{D}, 0}, \Pi_{\mathcal{D}}, \nabla_{\mathcal{D}}\right)$ be given by (5.4), (5.6) and (5.8), for some $\left(A_{K}\right)_{K \in \mathcal{M}}$. We take $\theta>0$ such that (5.14) and (5.15) hold.

Then there exists $\nu>0$ and $C_{15}$ only depending on $\Omega, p$ and $\theta$ such that, for all $\boldsymbol{\xi} \in \mathbb{R}^{d}, T_{\mathcal{D}}(\boldsymbol{\xi}) \leq C_{15}|\boldsymbol{\xi}|^{\nu}$.

\section{Proof}

Let $v \in X_{\mathcal{D}, 0}$. Noticing that, if $\sigma$ is an edge between $K$ and $L$, we have $\frac{\left|v_{K}-v_{L}\right|}{d_{K, \sigma}+d_{L, \sigma}} \leq \frac{\left|v_{K}-v_{\sigma}\right|}{d_{K, \sigma}}+\frac{\left|v_{L}-v_{\sigma}\right|}{d_{L, \sigma}}$, [20, Lemma 5.5] and (5.16) show that there exists $C_{16}$ only depending on $\Omega$ and $\theta$ such that

$$
\left\|\Pi_{\mathcal{D}} v(\cdot+\boldsymbol{\xi})-\Pi_{\mathcal{D}} v\right\|_{L^{1}\left(\mathbb{R}^{d}\right)} \leq \sqrt{d}|\boldsymbol{\xi}| \sum_{K \in \mathcal{M}} \sum_{\sigma \in \mathcal{E}_{K}}|\sigma| d_{K, \sigma}\left|\frac{v_{\sigma}-v_{K}}{d_{K, \sigma}}\right| \leq C_{16}|\boldsymbol{\xi}|\left\|\nabla_{\mathcal{D}} v\right\|_{L^{p}(\Omega)^{d}}
$$

Moreover, by [20, Lemma 5.4] and (5.16) we can find $r>p$ and $C_{17}$ only depending on $\Omega, p$ and $\theta$ such that

$$
\left\|\Pi_{\mathcal{D}} v\right\|_{L^{r}\left(\mathbb{R}^{d}\right)} \leq C_{17}\left\|\nabla_{\mathcal{D}} v\right\|_{L^{p}(\Omega)^{d}} .
$$

We now write, thanks to Hölder's inequality, $\|\cdot\|_{L^{p}\left(\mathbb{R}^{d}\right)} \leq\|\cdot\|_{L^{1}\left(\mathbb{R}^{d}\right)}^{\nu}\|\cdot\|_{L^{r}\left(\mathbb{R}^{d}\right)}^{1-\nu}$ where $\nu>0$ is such that $\frac{\nu}{1}+\frac{1-\nu}{r}=\frac{1}{p}$, and (5.29) and (5.30) conclude the proof.

\section{Appendix}

The proof of the following theorem is inspired by [24].

Theorem 5.13 (Discrete Aubin-Simon lemma) Let $T>0$ and let $B$ be a Banach space. Let $\left(B_{m}\right)_{m \in \mathbb{N}}$ be a sequence of finite dimensional subspaces of $B$. For any $m \in \mathbb{N}$, let $N_{m} \in \mathbb{N}^{*}, t_{m}^{(0)}=0<$ $t_{m}^{(1)}<\ldots<t_{m}^{\left(N_{m}\right)}=T$ and $\delta t_{m}^{\left(n+\frac{1}{2}\right)}=t_{m}^{(n+1)}-t_{m}^{(n)}, n=0, \ldots, N_{m}-1$. Let $\left\{v_{m}^{(n)}, n=0, \ldots, N_{m}\right\} \subset B_{m}$ and let $v_{m} \in L^{1}\left(0, T ; B_{m}\right)$ be defined, for a given real family $\left(\alpha_{m}^{(n)}\right)_{n=0, \ldots, N_{m}-1}$, by

$$
\begin{aligned}
& v_{m}(t)=\left(1-\alpha_{m}^{(n)}\right) v_{m}^{(n)}+\alpha_{m}^{(n)} v_{m}^{(n+1)} \in B_{m}, \\
& \text { for a.e. } t \in\left(t_{m}^{(n)}, t_{m}^{(n+1)}\right) \text { and } n \in\left\{0, \ldots N_{m}-1\right\} .
\end{aligned}
$$

Let $\delta_{m} v_{m}$ be the "discrete time derivative", defined by:

$$
\delta_{m} v_{m}(t)=\delta_{m}^{\left(n+\frac{1}{2}\right)} v_{m}:=\frac{1}{\delta_{m}^{\left(n+\frac{1}{2}\right)}}\left(v_{m}^{(n+1)}-v_{m}^{(n)}\right) \text { for a.e. } t \in\left(t_{m}^{(n)}, t_{m}^{(n+1)}\right) \text { and } n \in\left\{0, \ldots, N_{m}-1\right\} .
$$

Let $\|\cdot\|_{X_{m}}$ and $\|\cdot\|_{Y_{m}}$ be two norms on $B_{m}$. We denote by $X_{m}$ the space $B_{m}$ endowed with the norm $\|\cdot\|_{X_{m}}$ and by $Y_{m}$ the space $B_{m}$ endowed with the norm $\|\cdot\|_{Y_{m}}$. We assume that

(h1) For any sequence $\left(w_{m}\right)_{m \in \mathbb{N}}$ such that $w_{m} \in B_{m}$ and $\left(\left\|w_{m}\right\|_{X_{m}}\right)_{m \in \mathbb{N}}$ is bounded, there exists $w \in B$ such that, up to a subsequence, $w_{m} \rightarrow w$ in $B$ as $m \rightarrow+\infty$.

(h2) For any sequence $\left(w_{m}\right)_{m \in \mathbb{N}}$ such that $w_{m} \in B_{m},\left(\left\|w_{m}\right\|_{X_{m}}\right)_{m \in \mathbb{N}}$ is bounded, there exists $w \in B$ such that $w_{m} \rightarrow w$ in $B$ and $\left\|w_{m}\right\|_{Y_{m}} \rightarrow 0$ as $m \rightarrow+\infty$, we have $w=0$.

(h3) The family $\left(\alpha_{m}^{(n)}\right)_{n=0, \ldots, N_{m}-1, m \in \mathbb{N}}$ and the sequence $\left(\left\|v_{m}\right\|_{L^{1}\left(0, T ; X_{m}\right)}\right)_{m \in \mathbb{N}}$ are bounded.

(h4) The sequence $\left(\left\|\delta_{m} v_{m}\right\|_{L^{1}\left(0, T ; Y_{m}\right)}\right)_{m \in \mathbb{N}}$ is bounded.

Then there exists $v \in L^{1}(0, T ; B)$ such that, up to a subsequence, $v_{m} \rightarrow v$ in $L^{1}(0, T ; B)$ as $m \rightarrow+\infty$. 
Proof The first step is to apply [24, Lemma 3.2], which states that, under Hypothesis (h1), there exists $C_{X}>0$ such that, for all $m \in \mathbb{N}$, for all $v \in B_{m},\|v\|_{B} \leq C_{X}\|v\|_{X_{m}}$. Following the proof of [24, Lemma 3.1], thanks to (h1) and (h2) we remark that the following variant of Lions' lemma holds: for all $\varepsilon>0$ there exists $C(\varepsilon)>0$ such that

$$
\forall m \in \mathbb{N}, \forall v \in B_{m},\|v\|_{B} \leq \varepsilon\|v\|_{X_{m}}+C(\varepsilon)\|v\|_{Y_{m}}
$$

Let us now notice, using (h4), that there exists $C_{t}>0$ such that, $\forall m \in \mathbb{N}, \sum_{n=0}^{N_{m}-1} \delta t_{m}^{\left(n+\frac{1}{2}\right)}\left\|\delta_{m}^{\left(n+\frac{1}{2}\right)} v_{m}\right\|_{Y_{m}} \leq$ $C_{t}$. We therefore get from (h3) that the sequence $\left(\left\|v_{m}\right\|_{B V\left(0, T ; Y_{m}\right)}\right)_{m \in \mathbb{N}}$ is bounded since, denoting by $\widetilde{v}_{m}^{(n)}=\left(1-\alpha_{m}^{(n)}\right) v_{m}^{(n)}+\alpha_{m}^{(n)} v_{m}^{(n+1)}$ the value of $v_{m}(t)$ on $\left(t^{(n)}, t^{(n+1)}\right)$, we can write

$$
\begin{aligned}
\sum_{n=0}^{N_{m}-2}\left\|\widetilde{v}_{m}^{(n+1)}-\widetilde{v}_{m}^{(n)}\right\|_{Y_{m}} & \leq \sum_{n=0}^{N_{m}-2}\left(\left|\alpha_{m}^{(n+1)}\right| \delta t_{m}^{\left(n+\frac{3}{2}\right)}\left\|\delta_{m}^{\left(n+\frac{3}{2}\right)} v_{m}\right\|_{Y_{m}}+\left|1-\alpha_{m}^{(n)}\right| \delta t_{m}^{\left(n+\frac{1}{2}\right)}\left\|\delta_{m}^{\left(n+\frac{1}{2}\right)} v_{m}\right\|_{Y_{m}}\right) \\
& \leq\left(1+2 C_{\alpha}\right) C_{t},
\end{aligned}
$$

where $C_{\alpha}$ is a bound of the family $\left(\alpha_{m}^{(n)}\right)_{n=0, \ldots, N_{m}-1, m \in \mathbb{N}}$. We then extend $v_{m}$ by symmetry on $(-T, 2 T)$, setting $v_{m}(-t)=v_{m}(t)$ and $v_{m}(T+t)=v_{m}(T-t)$ for a.e. $t \in(0, T)$. We get $v_{m} \in B V\left(-T, 2 T ; Y_{m}\right)$ with $\left\|v_{m}\right\|_{B V\left(-T, 2 T ; Y_{m}\right)} \leq 3\left(1+2 C_{\alpha}\right) C_{t}$. We also see, using (h3), that the sequence $\left(\left\|v_{m}\right\|_{L^{1}\left(-T, 2 T ; X_{m}\right)}\right)_{m \in \mathbb{N}}$ is bounded, say by $C_{0}$. Applying (5.32), we write, for any $\tau \in(0, T)$ and $t \in(-T, 2 T-\tau)$,

$$
\left\|v_{m}(t+\tau)-v_{m}(t)\right\|_{B} \leq \varepsilon\left\|v_{m}(t+\tau)-v_{m}(t)\right\|_{X_{m}}+C(\varepsilon)\left\|v_{m}(t+\tau)-v_{m}(t)\right\|_{Y_{m}},
$$

which provides

$$
\int_{-T}^{2 T-\tau}\left\|v_{m}(t+\tau)-v_{m}(t)\right\|_{B} \mathrm{~d} t \leq 2 C_{0} \varepsilon+3 C(\varepsilon)\left(1+2 C_{\alpha}\right) C_{t} \tau .
$$

This proves that $\int_{-T}^{2 T-\tau}\left\|v_{m}(t+\tau)-v_{m}(t)\right\|_{B} \mathrm{~d} t$ tends to 0 with $\tau$, uniformly with respect to $m \in \mathbb{N}$. Multiplying $v_{m}$ by a function $\psi \in C_{c}^{\infty}(-T, 2 T)$ equal to 1 on $(0, T)$, we may then apply the compactness theorem [24, Theorem 2.1] to obtain the relative compactness of the family $\left(v_{m}\right)_{m \in \mathbb{N}}$ in $L^{1}(0, T ; B)$.

\section{References}

[1] I. Aavatsmark, T. Barkve, O. Boe, and T. Mannseth. Discretization on non-orthogonal, quadrilateral grids for inhomogeneous, anisotropic media. J. Comput. Phys., 127(1):2-14, 1996.

[2] B. Andreianov, F. Boyer, and F. Hubert. Discrete duality finite volume schemes for Leray-Lions-type elliptic problems on general 2D meshes. Num. Met. Part. Diff. Eq., 23(1):145-195, 2007.

[3] L. Beirão da Veiga, and G. Manzini, An a posteriori error estimator for the mimetic finite difference approximation of elliptic problems. Internat. J. Numer. Meth. Engrg. 76(11):1696-1723, 2008.

[4] L. Beirão da Veiga, J. Droniou, and G. Manzini, A unified approach to handle convection terms in Finite Volumes and Mimetic Discretization Methods for elliptic problems. IMA J. Numer. Anal., 31(4):1357-1401, 2011.

[5] F. Boyer, and F. Hubert. Finite volume method for 2D linear and nonlinear elliptic problems with discontinuities. SIAM Journal on Numerical Analysis, 46(6):3032-3070, 2008.

[6] E. Burman, and A. Ern. Discontinuous Galerkin approximation with discrete variational principle for the nonlinear Laplacian. C. R. Acad. Sci. Paris, Ser. I, 346(17):1013-1016, 2008.

[7] F. Brezzi, K. Lipnikov, and V. Simoncini. A family of mimetic finite difference methods on polygonal and polyhedral meshes. Math. Models Methods Appl. Sci., 15(10):1533-1551, 2005. 
[8] F. Brezzi, K. Lipnikov, and M. Shashkov. Convergence of the mimetic finite difference method for diffusion problems on polyhedral meshes. SIAM J. Numer. Anal., 43(5):1872-1896, 2005.

[9] F. Brezzi, K. Lipnikov, and M. Shashkov. Convergence of mimetic finite difference method for diffusion problems on polyhedral meshes with curved faces. Math. Models Methods Appl. Sci., 16(2): 275-297, 2006.

[10] F. Catté, P. L. Lions, J. M. Morel, and T. Coll. Image selective smoothing and edge detection by nonlinear diffusion. SIAM J. Num. Anal., 29:182-193, 1992.

[11] C. Chainais-Hillairet and J. Droniou. Convergence analysis of a mixed finite volume scheme for an elliptic-parabolic system modeling miscible fluid flows in porous media. SIAM J. Numer. Anal., 45(5):2228-2258, 2007.

[12] K. Deimling. Nonlinear functional analysis. Springer-Verlag, Berlin, 1985.

[13] K. Domelevo and P. Omnes. A finite volume method for the Laplace equation on almost arbitrary two-dimensional grids. M2AN Math. Model. Numer. Anal., 39(6):1203-1249, 2005.

[14] O. Drblíková and K. Mikula. Convergence analysis of finite volume scheme for nonlinear tensor anisotropic diffusion in image processing. SIAM J. Numer. Anal., 46(1):37-60, 2007/08.

[15] J. Droniou. Finite volume schemes for fully non-linear elliptic equations in divergence form. M2AN Math. Model. Numer. Anal., 40(6):1069-1100, 2006.

[16] J. Droniou and R. Eymard. A mixed finite volume scheme for anisotropic diffusion problems on any grid. Numer. Math., 105(1):35-71, 2006.

[17] J. Droniou and R. Eymard. Study of the mixed finite volume method for Stokes and Navier-Stokes equations. Numer. Meth. P. D. E., 25(1):137-171, 2009.

[18] J. Droniou, R. Eymard, T. Gallouët, and R. Herbin. A unified approach to mimetic finite difference, hybrid finite volume and mixed finite volume methods. Math. Models Methods Appl. Sci., 20(2):265$295,2010$.

[19] R. Eymard, T. Gallouët, and R. Herbin. Cell centred discretisation of non linear elliptic problems on general multidimensional polyhedral grids. J. Numer. Math., 17(3):173-193, 2009.

[20] R. Eymard, T. Gallouët, and R. Herbin, Discretization of heterogeneous and anisotropic diffusion problems on general nonconforming meshes, SUSHI: a scheme using stabilization and hybrid interfaces. IMA J. Numer. Anal., 30(4):1009-1043, 2010.

[21] R. Eymard, C. Guichard, and R. Herbin. Small-stencil 3D schemes for diffusive flows in porous media. M2AN Math. Model. Numer. Anal., 46(2):265-290, 2012.

[22] R. Eymard, A. Handlovičová, R. Herbin, K. Mikula and O. Stašová. Applications of approximate gradient schemes for nonlinear parabolic equations. submitted, 2012.

[23] R. Eymard and R. Herbin. Gradient Scheme Approximations for Diffusion Problems. In Finite volumes for complex applications VI, Problems $\mathcal{E} 3$ Perspectives, 1:439-447. Springer, Berlin, 2011.

[24] T. Gallouët and J.-C. Latché. Compactness of discrete approximate solutions to parabolic PDEs application to a turbulence model. To appear in Comm. on Pure and Applied Analysis, 2012.

[25] F. Hermeline. Approximation of diffusion operators with discontinuous tensor coefficients on distorted meshes. Comput. Methods Appl. Mech. Engrg., 192(16-18):1939-1959, 2003.

[26] J. Leray and J.-L. Lions. Quelques résultats de Višik sur les problèmes elliptiques nonlinéaires par les méthodes de Minty-Browder. Bull. Soc. Math. France, 93:97-107, 1965. 
[27] G. J. Minty: On a monotonicity method for the solution of non- linear equations in Banach spaces. Proc. Nat. Acad. Sci. U.S.A., 50:1038-1041, 1963.

[28] G. Strang. Variational crimes in the finite element method. In The mathematical foundations of the finite element method with applications to partial differential equations (Proc. Sympos., Univ. Maryland, Baltimore, Md., 1972), pages 689-710. Academic Press, New York, 1972.

[29] J. Weickert. Coherence-enhancing diffusion filtering. Int. J. Comput. Vision, 31:111-127, 1999. 